\title{
Nondestructive Damage Assessment of Composite Structures Based on Wavelet Analysis of Modal Curvatures: State-of-the-Art Review and Description of Wavelet-Based Damage Assessment Benchmark
}

\author{
Andrzej Katunin \\ Institute of Fundamentals of Machinery Design, Silesian University of Technology, 18A Konarskiego Street, 44-100 Gliwice, Poland \\ Correspondence should be addressed to Andrzej Katunin; andrzej.katunin@polsl.pl
}

Received 13 February 2015; Revised 6 June 2015; Accepted 11 June 2015

Academic Editor: Roger Serra

Copyright (C) 2015 Andrzej Katunin. This is an open access article distributed under the Creative Commons Attribution License, which permits unrestricted use, distribution, and reproduction in any medium, provided the original work is properly cited.

\begin{abstract}
The application of composite structures as elements of machines and vehicles working under various operational conditions causes degradation and occurrence of damage. Considering that composites are often used for responsible elements, for example, parts of aircrafts and other vehicles, it is extremely important to maintain them properly and detect, localize, and identify the damage occurring during their operation in possible early stage of its development. From a great variety of nondestructive testing methods developed to date, the vibration-based methods seem to be ones of the least expensive and simultaneously effective with appropriate processing of measurement data. Over the last decades a great popularity of vibration-based structural testing has been gained by wavelet analysis due to its high sensitivity to a damage. This paper presents an overview of results of numerous researchers working in the area of vibration-based damage assessment supported by the wavelet analysis and the detailed description of the Waveletbased Structural Damage Assessment (WavStructDamAs) Benchmark, which summarizes the author's 5-year research in this area. The benchmark covers example problems of damage identification in various composite structures with various damage types using numerous wavelet transforms and supporting tools. The benchmark is openly available and allows performing the analysis on the example problems as well as on its own problems using available analysis tools.
\end{abstract}

\section{Introduction}

Increasing demands to integrity and safety of composite structural elements being in operation in aircrafts and other vehicles have induced the development of nondestructive testing (NDT) methods, which should be sensitive to various types of damage occurring in composites considering the specificity of their different internal structures and the specificity of damage initiation and propagation. These methods should also fulfill additional criteria; for example, they should allow for early damage detection and identification; they should be reference-free (i.e., the conclusion about the damage presence and its type and shape should be determined based only on results of inspection of a damage structure without necessity of its comparison with a healthy structure or a model); they should allow performing the testing procedures in environmental conditions and be inexpensive.
From a great variety of NDT methods developed to date, a group of methods based on vibration testing with advanced processing of measured signals seem to fulfill the abovementioned criteria and can be successfully used both for laboratory tests and in industrial studies. The acquisition of experimental data is usually based on the modal analysis. The classical modal analysis, that is, the analysis of changes in natural frequencies and modal shapes when the tested structure is excited to vibration by an external source, can be considered only if a damage is sufficiently large, since the analysis of modal parameters (natural frequencies, damping ratios) is global and thus there is no possibility to locate damage. Moreover, such approach is not referencefree. Considering additional factors, for example, the great variability of mechanical properties of composite structures, the testing approach which is based on evaluation of changes in natural frequencies of a tested structure is not effective in 
engineering applications. An extended analysis of vibrationbased NDT methods can be found, for example, in [1], where the authors presented an overview on methods based on evaluation of natural frequencies and frequency response functions (FRFs), like modal assurance criterion or frequency domain assurance criterion.

A great potential of vibration-based NDT lies in the analysis of modal shapes of vibrations. Usually the resonant shapes are taken into consideration; however it is not a necessary condition for such analysis. The idea of damage assessment based on modal shapes is that a damage occurring in a tested structure causes local changes in its stiffness; the modal shapes reflect such changes much better than natural frequencies. Several studies on analysis of curvature of modal shapes can be found in available literature $[1,2]$. Ho and Ewins [3] used various measures, like flexibility index, mode shape curvature, or mode shape amplitude comparison, in order to localize damage by analyzing modal shapes. Their approaches reveal comparatively good results for detection and localization of damage sites of medium and great extent. The authors used differentiation of modal shapes in some of their measures, which is quite effective, especially in the case when the higher-order derivatives are considered. However, the local changes in modal shapes depend on size of damage and in case of small damage these changes are not recognizable. In order to detect, localize, and identify the damage properly, advanced signal processing procedures are necessary. Several approaches have been applied in such problems to date. A simple and quite effective approach is based on higher-order derivatives of modal shapes of vibration. Although this approach has numerous disadvantages such as error propagation and amplification, quite low sensitivity, and difficulties in results interpretation, it can be successfully used in numerous applications. Several attempts in this area were presented in [4-9]. In order to improve the sensitivity of this approach to structural damage detection, various techniques have been applied to date. The approach originated by Ratcliffe [10] was based on application of modified Laplacian operator on modal shapes of vibration, which allows for detection and localization of structural damage. The authors of [11] have developed the curvature damage factor, which indicates the nodal position of a damage based on comparison of healthy and damaged structure. An original algorithm of precise detection and localization of damage in beams was proposed by Chandrashekhar and Ganguli [12]. Another interesting approach was presented by Sazonov and Klinkhachorn [13], where the authors used strain energy mode shapes in order to detect and localize damage. This approach reveals relatively high sensitivity and accuracy in damage detection and localization. The authors of [14] used a smoothing method for damage detection and localization of a 2D structure. Further studies on enhancing the sensitivity and accuracy of damage detection and localization cover optimization-based approach [15], Hilbert-Huang transform [16], and many others. In order to improve detectability of damage in structures, several studies were performed using modal updating techniques. In this case, modal parameters (natural frequencies and modal shapes) of a tested structure are used for construction of analytical or numerical model of this structure and then the resulting model is compared to damaged structure. Therefore, the model updating approach allows for reduction of inaccuracies in a model by its updating using experimental data obtained from the tested object. It also has an additional advantage-a possibility of prediction of structural response without necessity of performing additional experiments. Several model updating techniques were developed. One should pay attention to two surveys on these techniques $[17,18]$ that describe numerous approaches used for model updating, both iterative and noniterative. A comparative study on model updating techniques using certain quality indices based on natural frequencies, modal shapes, and FRFs was described in [19]. Recent studies in model updating techniques used various approaches, including perturbation methods [20], Zernike moment descriptors $[21,22]$, and others. Due to the applied pointwise comparison of modal shapes, it is possible to localize a damage with relatively high accuracy.

One of the most promising approaches used for structural damage identification (SDI) in composites is based on wavelet analysis. This approach involves application of wavelet transform to modal shapes of vibration. It has gained a great popularity over the last decade mainly due to the outstanding sensitivity and ability of identification of abrupt changes in modal shapes caused by local changes of stiffness of a tested structure. The fundamentals on singularity detection can be found in [23]. Usually, two general types of wavelet transforms are applied in vibration-based SDI problems, namely, continuous wavelet transform (CWT) and discrete wavelet transform (DWT). The main difference between CWT and DWT is that CWT is related with shifting and scaling of a wavelet over a continuum of its parameters, while in DWT a wavelet is shifted and scaled over a discrete set of values. Usually, these operations are performed over the set of powers of 2. As a result, from CWT-based analysis, one obtains a scalogram (a matrix of coefficients which represent signal energy) over the space (dimensions of a signal) and continuously changed scale. The wavelet coefficients in a scalogram represent degree of matching of an applied wavelet to an analyzed signal at different scales and locations. In contrast to CWT, DWT operates on discrete values of shifts and scales, which, considering Mallat's multiresolution analysis (see [23] for details), can be described as hierarchical filtering procedure of a signal using a set of low-pass and high-pass filters, related to scaling and wavelet functions, respectively. In the case of $1 \mathrm{D}$ DWT, this results in two sets of coefficients at each level of decomposition: the sets approximation and details coefficients. The number of coefficients in these sets is two times smaller than an original signal due to the decimation procedure which selects only the even indexed samples from the decomposed signal before filtering procedure. Considering that DWT operates only on discrete set of values, this transform is much more computationally efficient with respect to CWT. One of the modifications of DWT is a stationary wavelet transform (SWT), where the decimation procedure is omitted. This causes SWT to be highly redundant with respect of DWT. At the expense of redundancy, SWT allows for near shift invariant analysis which is a useful property in SDI problems that improves an 
accuracy of damage localization. The detailed description and comparison between DWT, SWT, and some other wavelet transforms (that also found an application in SDI problems) are presented in [24].

The first published paper on application of wavelet analysis for SDI was prepared by Surace and Ruotolo [25], where they used CWT for detection of damage in beam structures. The studies on application of spatial version of CWT with Haar wavelets for SDI of cracks in beams were presented in $[26,27]$. Further studies were focused on selection of appropriate parameters of the analysis, for example, the wavelet, its order, and a scale parameter of CWT. In [28], the authors applied spatial CWT with Gabor wavelets for SDI in Timoshenko beams based on numerical data. By handling the scale parameters it was possible to detect and precisely localize the modelled crack in the beam. Further, the authors generalized their approach for 2D structures and performed the studies of estimation of damage position and its relative depth $[29,30]$. They show a great effectiveness of Gabor wavelets in SDI problems using the wavelet analysis. The authors of [31] used CWT-based algorithm with the Lipschitz exponent estimation for damage detection and localization in beam structures based on numerical data. On the basis of the obtained values of Lipschitz exponent, the authors performed an analysis of relative crack depth estimation with use of Mexican hat wavelet. Several studies using CWT-based approach were also performed by the Greek scientific group. The researchers from this group studied the influence of applied wavelet during SDI in beams [32] and found that it has a great impact on sensitivity and thus detectability of damage. They also performed experimental validation of CWT-based approach on beam-like [32] and plate-like structures [33, 34] using symlets. Similar approach has been developed by Gentile and Messina [35], who performed SDI in beams using Gaussian wavelets. They also investigated the influence of the boundary conditions of beams and the presence of noise on the sensitivity of the proposed method. The studies on application of CWT-based approach in SDI problems were also performed by the Polish scientific group. Rucka and Wilde performed preliminary studies on SDI in damaged Plexiglas plate using spatial CWT-based approach [36]. Further, they presented results of SDI based on static deflections of beams [37] and vibrated beams and plates [38]. They also presented a comparative study of various wavelets applicable to the investigated problem with respect to specific parameters of wavelets: number of vanishing moments, symmetry, and a width of the effective support. Based on this comparative study they selected Gaussian and reversed biorthogonal wavelets as the most effective for the CWT-based damage identification. Results of other researchers [39] confirmed that the Gaussian wavelets are probably the most effective ones in SDI problems when CWT-based approach is used. This is mathematically justified, since the Gaussian wavelets are regular, infinitely derivable, and perfectly local in space and frequency domains; that is, they achieve the minimum spacefrequency product from Heisenberg uncertainty principle.

Additional approach of wavelet-based SDI developed by Zhong and Oyadiji uses SWT. This transform has several limitations with respect to CWT (e.g., orthogonality and a compact support of applied wavelets); however it is more computationally efficient and more accurate in SDI problems than CWT. The authors applied their SWT-based algorithm for detection and localization of cracks in simply supported beams [40] with high accuracy. The authors stated that their approach had better detection and localization abilities than CWT-based algorithms and the algorithms based on DWT. The comparison was performed for the analyses based on symlet of order 4 .

Several studies were also performed using DWT-based approach. Probably the first application of this approach to SDI in composites was presented by Sung et al. [41], where the authors used DWT for detection of low-velocity impact damage. Then, DWT-based approach has not been developed except the hybrid SDI algorithm of combination of CWT and DWT proposed by the authors of [42]. The first attempts in application of DWT-based algorithms were made by the author of this paper. Several experimental studies were performed on cantilever composite beams with single and multiple damage sites using DWT and B-spline wavelets [43]. Then, the approach was extended to $2 \mathrm{D}$ problems and verified both on numerical as well as on experimental modal shapes of vibration of predamaged composite plates [44]. Although DWT has several limitations with respect to CWT (same as for SWT-CWT, mentioned above), CWT and SWT are redundant with respect to DWT. The results of comparative analyses performed for the mentioned transforms and a lifting wavelet transform (LWT) have proved that DWT is the most effective transform in SDI problems [45]. The comparative studies also cover the estimation of the effectiveness of particular wavelets applied for SDI of numerical modal shapes of a rotor blade with two locations of cracks. The DWT-based SDI algorithm was also adapted to the $2 \mathrm{D}$ problems in polar coordinates [46]; the analyses were performed on numerical modal shapes of composite circular plates clamped on the edge.

Recently, the improvement of wavelet-based SDI algorithms has been based on adaptation novel transforms and hybridization of the existing ones with supporting computational methods including the approaches based on softcomputing and artificial intelligence. An effective approach in SDI problems was proposed by Bagheri et al. [47], who used a discrete curvelet transform in order to detect a crack-like surface structural damage. Several studies on improvement of SDI algorithm were performed by the author's team. The novel approaches in wavelet analysis were adapted for SDI problems; for example, the discrete multiwavelet transform (DMWT) [48], quaternion wavelet transform [49] (HWT), and quincunx wavelet transform (QWT) [50] were used for damage identification in composite structures. Considering the great properties and results obtained by Unser and Blu [51], who originated the fractional discrete wavelet transform (FrDWT), this approach was adapted to SDI problems. The first studies were performed on numerical modal shapes of cantilever composite beams [52] and obtained results revealed a significant improvement with respect to previously applied algorithms. Further, the algorithm was extended for 2D SDI problems based on the transform developed by Chaudhury and Unser [53] and applied for identification of 
various types of damage in composite structures [54, 55]. Finally, the modification of mentioned algorithm for an improvement of resolution of resulting sets of coefficients, and thus accuracy of damage localization, was proposed [56].

Several authors combined the wavelet-based algorithms with artificial neural networks (ANN) for improvement the detection and localization ability. Yam et al. [57] combined wavelet-based algorithm with ANN for improving the damage identification in sandwich structures; Rucka and Wilde [58] proposed a combination of wavelet transform with ANN for improvement of identification ability of structural damage, while Hein and Feklistova [59] used such a combination for estimation of damage indices, which describe the delamination occurrence in composite structures. An original approach of combination of CWT with ANN was proposed by Morlier et al. [60], where CWT was used for damage localization, while ANN was used for its identification using a simulated database. In several studies the optimization procedures were used in order to enhance the sensitivity, detectability, and identification ability of damage in structures. An interesting algorithm was presented by the authors of [61], where they combined wavelet-based approach with Bayesian networks for the damage identification in composite structures. Another approach assumes the hybridization of wavelet-based techniques with optimization procedures, which were used for improvement of the sensitivity and localization ability of damage. The developed method presented by the authors of [62] is based on 2D wavelet transform, which allows for damage detection and localization and then the performed particle swarm optimization procedure evaluates the damage severity. The author's team presented several attempts in the field of hybrid SDI algorithms. The first study is concerned with detection and localization of delaminations in composite laminated beams based on the analysis of modal shapes obtained from numerical experiment [63]. The SDI algorithm is based on FrDWT with optimization of wavelets parameters, namely, the fractional order and a shift parameter, which allows for selection of a wavelet, which gives the best parameters for damage identification procedure. Further, this approach was extended to 2D SDI problems [54], where the common optimal parameters of applied wavelet were determined for a set of considered problems. In this case, surface damage in clamped laminated composite plates with various sizes and orientations was under investigation. The algorithm was verified on both numerical and experimental modal shapes of vibration. Finally, the metaoptimization procedure for SDI was proposed [64]. This approach allows obtaining a front of optimal nondominated solutions regarding the wavelet parameters and simultaneously lowering computational time without loss of accuracy.

Considering the overview presented above, one can highlight several problems and factors, which have a significant influence on the sensitivity of wavelet-based methods to a damage presence and accuracy of its localization. These factors are discussed in detail in the next section. Taking into account gained experience and obtained results in SDI problems, it was decided to prepare a benchmark which consists of SDI example problems and necessary tools for performing the analyses using wavelet-based methods. The benchmark and the benchmark example problems are described in detail in a separate section.

\section{Factors Influence on the Effectiveness of Structural Damage Assessment}

Though SDI based on modal analysis and wavelet-based processing of modal data has proven facilities in practical applications, several important factors have an influence on the detectability and localization accuracy of SDI. In spite of great sensitivity of wavelet-based algorithms on abrupt changes in analyzed signals, all of them are very sensitive to noise. Therefore, appropriate measurement conditions should be fulfilled in order to minimize an influence of noise on the resulting modal shapes. On the other hand, if the noise minimization is not possible or if it is not sufficient for the investigated case, the appropriate denoising procedures should be applied in the preprocessing step of the analysis. The second group of factors is connected with selection of adequate wavelet transform and the wavelets applied to the analysis, which are the deciding factors. Finally, the third group of important factors consists of problems with damage location, number of considered measurement points, and the boundary effect. These factors are analyzed in detail in the following section.

2.1. Measurement Conditions and Noise. One of the preliminary used measurement setups in SDI problems was based on external excitation of a tested structure and performing the measurements using accelerometers. The Greek scientific group mentioned previously used such a setup in their experimental studies [32-34], where the tested structures were excited by the electromagnetic vibrator and the measurements were performed using two accelerometers: one for performing the measurements of structural vibration response and another used as a reference. Such approach can be effective when the mass of accelerometers is negligibly small with respect to the mass of a tested structure; otherwise the presence of accelerometers on a surface of a tested structure may significantly bias the vibration response of a structure. This may cause hiding of the information about damage presence and location. Nevertheless, if such a setup is selected for testing, one should pay attention to two of the most important factors: the appropriate frequency range of an applied accelerometer and a type of accelerometer mounted to a tested structure in order to avoid additional signal disturbances and measurement noise. These as well as other factors which influence measurement accuracy (cable connections requirements, environmental temperature, electrical inference, etc.) were discussed in detail in [65]. An example of advanced practical application of vibration measurements by accelerometers were presented by Smith et al. [66], where SDI was performed on F-15 tactical fighter plane structure and then processed by wavelet-based technique in order to detect structural damage.

Significant improvement of a quality of measured vibration signals was reached when the laser Doppler vibrometers (LDV) began to be applied in the SDI test setups. The general principle of LDV is based on extraction of the information 
about frequency and magnitude of vibration of a tested surface in a specific point from the Doppler shift of a reflected laser beam. Since this measurement device provides noncontact measurements using a laser beam, the signal acquisition is very accurate with simultaneous elimination of influence of measurement device on a tested structure. The LDVs are widely used for SDI problems for test setups both by the author $[44,49,50,55]$ and by other researchers [39, 67-74]. The crucial importance during such measurements has the appropriate reflectivity of a surface of a tested structure, what is directly connected with quality of acquired signals. For this purpose the reflective tape should be adhered on the surface or the surface should be covered by the antiglare powder.

In order to avoid signal disturbances, they can be denoised in the preprocessing stage; however setting inappropriate filtering parameters may cause the significant damage features to be filtered out together with the noise. Several studies were performed in order to investigate this problem. The authors of [32] investigated the influence of artificially added noise of $1 \%$ with respect to the magnitudes of modal shapes of a tested structure and show that the detectability of damage becomes much worse and when the noise magnitude reaches $5 \%$ the damage detection is impossible using the applied algorithm. The same scientific group performed the analysis of dependence of detectability of cracks with various depths under variable signal-to-noise ratios (SNRs). The analysis shows that crack is fully detectable only in the noiseless case (SNR: 90-100 dB); in the case when SNR equals $60-70 \mathrm{~dB}$, only the cracks with a depth higher than $20 \%$ of a total thickness of a tested structure can be detected and when SNR equals 50-60 dB, the cracks with a depth up to $40 \%$ of a total thickness remain undetectable following the proposed procedure. The authors of [37] stated that the noise does not corrupt the measurement signals; however the damage depth was relatively large and equalled $25 \%$ of a total thickness of a tested structure. Zhong and Oyadiji [40] achieved the damage detectability with a noise of up to $5 \%$ with respect to the magnitudes of modal shapes and with a crack depth of $5 \%$ of total thickness; however their method uses a baseline obtained from the cubic curve fitting of modal shapes. The author's studies on SNR level [45] show that the crack with a depth of $8.3 \%$ of total thickness is still detectable when SNR is equal to $55 \mathrm{~dB}$. For example, SNR for LDV in typical measurement conditions (distance to the sample of $1 \mathrm{~m}$ ) is $65.4 \mathrm{~dB}$ [75], which allows for precise identification of even small damage.

Another testing approach is based on performing the measurements using embedded measurement devices (e.g., embedded piezoelectric patches and transducers (PZTs) and fiber Bragg grating sensors (FBGs)), which are capable not only of SDI but also of continuous structural health monitoring (SHM). An advantage of PZTs is that they can be used as both actuators and sensors which is desired property in SHM systems. Damage identification procedure using PZTs is based on observation of character of propagation of Lamb waves in a tested structure; a possible damage disturbs these waves which is an indicator of damage presence and its location. When a damage causes small structural changes in a tested structure, advanced signal processing techniques are necessary for its detection and localization. Several damage localization techniques using PZT-based measurements with further processing of measured signals were presented by Ostachowicz et al. [76]. Among other signal processing techniques, the wavelet transform is one of the most attractive ones for this purpose due to its very high sensitivity to local singularities in signals. Therefore, it found an application in PZT-based SDI in numerous studies. The authors of [77, 78] used CWT and DWT for denoising of measurement signals. The application of wavelet-based data processing in PZT-based experimental studies can be found in [57, 7982]. Another approach, which included use of a distributed sensor network and wavelet-based analysis, was described by Staszewski [83] and Huang et al. [84]. The authors of [85] proposed the measurement system based on the FBG sensors. The testing procedure assumed further evaluation of structural condition using DWT-based algorithm. Lu et al. [86] used FBG sensors for impact damage detection and localization in composite structures, where the wavelet transform was used in the preprocessing step for denoising of measurement signals. The embedded measurement systems allow for the displacement measurements with a very high precision; however it is suitable to use them for SHM, because a tested structure should be supplied with such systems at the stage of its manufacturing.

Several attempts in analysis of noisy data in SDI problems were made by Cao et al. [72-74]. They proposed the SDI algorithm based on Teager energy operator (TEO) combined with CWT, which ensures damage detectability and its localization even if the acquired signals are biased by noise. The concept of TEO is also developed by Li et al. [87], who successfully applied gapped smoothing method and TEO for damage localization in composite lattice truss core sandwich structures.

\subsection{Selection of Wavelet Transform and Wavelets. Many SDI} studies with various wavelet transforms and wavelets have been performed in the last decade by numerous researchers. As it can be seen from the Introduction, the first application of wavelet analysis to SDI was performed using CWTbased algorithms. The effectiveness of the analysis, inter alia the damage detectability, strongly depends on the selected wavelet; therefore such an analysis should be performed for both the wavelet transform and selected wavelet. The first studies on application of wavelet analysis in SDI problems were based on the empirical observations of the resulting sets of coefficients after the transform. The authors [27, 88] used Haar wavelet for damage detection and localization; the authors of [89] used biorthogonal wavelets, while the authors of $[27,29,88]$ applied complex-valued Gabor wavelets for the analysis. The first systematic studies in this area were introduced by Hong et al. [31] and Douka et al. [32], where the authors proposed dependence between the number of vanishing moments of an applied wavelet and effectiveness of damage detection. Hong et al. applied Mexican hat wavelet, while Douka et al. [32-34] as well as Zhong and Oyadiji [40] used symlets in their studies. Further systematic study was presented by Rucka and Wilde [38], where the authors considered two parameters during selection of a wavelet for the transform: the number of vanishing moments and 
the length of effective support of the selected wavelet. The authors of [38] compared the considered modal shapes to 4th-order polynomial and selected the Gaussian wavelet as the best candidate for 1D SDI problem; for the investigated 2D problem they selected reversed biorthogonal wavelet of order 5.5. The Gaussian wavelets were also applied by Gentile and Messina in their studies for 1D SDI problem [35] and by Fan and Qiao [39] for 2D SDI problem.

In contrast to CWT, the SWT has some limitations to wavelets, which can be applied for the analysis; that is, the wavelet should be (semi-, bi-)orthogonal or orthonormal and should have a compact support. As the main contributors of the SWT-based algorithms for SDI problems stated [40], SWT is redundant with respect to CWT and DWT; however it allows for shift-invariant wavelet decomposition. For this purpose this type of wavelet transform was selected for SDI in beams in mentioned works as well as in further studies of the authors [90].

The application of DWT in SDI problems has been developed mainly by the author of the following paper. DWT also has limitations to the applicable wavelets, similar to SWT. Since the Gaussian wavelets proposed by the authors which used CWT-based algorithms are not applicable in the case of DWT-based analysis, the B-spline wavelets can be used as a substitute. From the comparative analyses presented in [44, 45], the DWT-based algorithm with B-spline wavelets was selected as the best solution for SDI problems due to the highest sensitivity of B-spline wavelets and the best computational efficiency of DWT. In the systematic analysis, two parameters selected by Rucka and Wilde [38] (the number of vanishing moments and the length of effective support of a wavelet) and additional parameter-symmetry of a wavelet-were considered. During selection of wavelet order, a compromise between the length of effective support and a number of vanishing moments of the wavelet should be reached, since greater length of effective support affects disturbances on the boundaries of damage and causes blurring of the damage coefficients. On the other hand, if the order of wavelet is too low a damage may not be detected.

In order to improve the detectability and accuracy of localization, the fractional B-spline wavelets were applied for SDI problems instead of integer-valued B-spline wavelets. This gives a possibility to select the wavelet order from the range of reals, which significantly improved the damage detectability and clearness of its position [53]. Moreover, the fractional B-spline wavelets are shift-invariant [51], which additionally improves the localization ability of a damage. In order to avoid the necessity of selection of the wavelet parameters, the hybrid algorithms with optimization of these parameters have been developed. These algorithms were applied both for 1D [63] and for 2D [54] SDI problems and allow for selection of optimal parameters for the applied wavelet. The multiobjective optimization function in these algorithms covered maximization of wavelet coefficients in damage location with simultaneous minimization of noise out of a damaged region. Finally, in order to automate the method and reduce the computation time of optimization routines, the metaoptimization algorithm was proposed for SDI problems [64], which is based on optimization of wavelet parameters as well as optimization of parameters of the optimization algorithm used for selection of optimal wavelet parameters. The algorithm was tested on both numerical and experimental data. Additional studies were addressed to the circular-shaped structures. In [46], the 2D DWT was adapted to polar coordinates and several numerical tests were presented. In [49] the quaternion wavelet transform was applied in the analysis of a composite circular-shaped plate with an inclusion. The advantages of dual-tree decomposition and its effectiveness in SDI problems are discussed in this section.

The initial studies in SDI in composites were performed using nonseparable wavelets [49] and multiwavelets [48]. The advantage of application of nonseparable wavelets (e.g., quincunx wavelets developed by the authors of [91]) is that the applied quincunx scheme [50] allows for finer scale progression than the dyadic scheme used in DWT. Moreover, quincunx-based scheme results in a single set of approximation and a single set of detail coefficients, which are additionally direction-invariant (in DWT the three sets of detail coefficients have preferred directions, where the diagonal one does not have a straightforward interpretation [92]). Application of multiwavelets originated by Strela [93] allows for better localization and reducing the unwanted artefacts in resulting coefficients [48]. Due to the fact that multiwavelets contain vectors of at least two scaling and wavelet functions, it creates a possibility of selection of a set of appropriate wavelets in the multiwavelet vectors of functions, which exactly match the necessities of the investigated problem. The application of nonseparable wavelets and multiwavelets creates new directions of research in the area, namely, of improvement sensitivity and localization ability of waveletbased algorithms applied in SDI problems.

\subsection{Damage Location, Sampling Distance, and Boundary} Effect. The location of a damage has a great influence on its detectability and ability of its localization. This is because the magnitudes of resulting coefficients are connected with the magnitudes of the analyzed modal shapes; that is, the higher magnitude in the given point of a damaged region the higher values of resulting coefficients after wavelet decomposition. Therefore, if the damage is located, for example, near the clamp of a tested structure, the resulting coefficients do not achieve the highest magnitudes and the damage detection is then problematic. Similar situation may happen if the damage location coincides with the node of modal shape, where the magnitudes of displacements are very low. Assuming that the damage position is unknown, the method should be sensitive to damage in every possible position. In order to avoid this problem, it is suitable to consider several modal shapes in the analysis, which increases a probability that the possible damage coincides with the local extremum of modal shapes. For this purpose the obtained coefficients can be added up. For avoiding the situation when positive and negative coefficients are reduced, the addition of their absolute values is suitable. In some cases the emphasizing of damaged coefficients is possible by adding the squared coefficients or by determining the Euclidean norm of coefficients for considered modal shapes. This technique is called the isotropic wavelet analysis. 
It was applied both in CWT-based algorithms [94] and in DWT-based ones [44, 45, 49, 50, 55].

Besides the factor discussed above, another important factor has an influence on the detectability of a damage and accuracy of its localization. The preliminary studies on influence of sampling distance on effectiveness of wavelet-based methods in SDI problems were established by Hong et al. in [31] and Douka et al. in [32]. It is obvious that the increase of number of measurement points (decrease of sampling distance) influences positively the damage detectability and accuracy in determining its location; however in experimental studies the increase of the number of measurement points causes a significant increase of testing duration, especially in the case of $2 \mathrm{D}$ problems. To overcome the problem of time-consuming testing, the authors of $[31,32]$ proposed an oversampling of measured data by application of cubic spline interpolation. This technique was further used by the authors of $[38,39,42]$. However, the application of any kind of oversampling may result in masking of the important information about the damage condition. Systematic studies on sampling distance were performed by Sazonov and Klinkhachorn [13], where the authors proposed the formulas to calculate the optimal sampling distance for various configurations of boundary conditions of beams, which minimize the effects of measurement noise and truncation errors occurring during undersampling or oversampling. The problem was also investigated by Zhong and Oyadiji [40]; however they evaluated the influence of sampling distance on quality of damage localization based on empirical observations only. They observed that decrease of a sampling distance results in increase of localization accuracy as well as magnitude of coefficients in the location of damage with respect to the rest of coefficients; thus, the detectability of small damage sites is also improved.

The last factor discussed in this paper is the boundary effect. This effect occurs by the specificity of application of wavelet analysis; namely, the analyzed signals always have a finite length and during shifting of the wavelet along the signal (considering that the applied wavelet has two or more vanishing moments) zero values outside the signal domain are added. As a result, when the wavelet reaches a region where the abrupt change in a signal occurs (from nonzero to zero values), obtained coefficients in this region become large. The number of biased coefficients in such a way is proportional to the number of vanishing moments of the applied wavelet. Additionally, the boundary effect depends on the boundary conditions of a tested structure. From the practical point of view, the boundary effect causes the regions near the boundaries in a tested structure to be inappropriate for analysis and they should not be considered further. The authors of [39] recommended neglecting the regions, where the boundary effect occurs. However, in the case of a small grid of measurement points, neglecting of the regions where the boundary effect occurs may significantly reduce the useful information. Moreover, when the boundary effect appears, the values on the boundaries are often much greater than the values in the damaged region; therefore it is suitable to reduce this effect also for the visualization purposes. In order to reduce the boundary effect, several approaches were established. The simplest and the most common approach is based on signal extension using various techniques like zero-padding and constant-padding (extension of a signal by adding zeros or constant values) and symmetric and antisymmetric reflection and periodization [95]. Loutridis et al. [34] recommended the reflection technique as suitable one for reducing the boundary effect in 2D SDI problems. More advanced methods of the boundary effect reduction were proposed by Rucka and Wilde $[37,38]$. The authors of these papers proposed the application of cubic spline interpolation of neighbor measurement points, which are much more effective in practice than previously discussed techniques. These methods are based on signal extension; however, they do not allow for proper damage detection and identification in the boundary regions of a tested structure. An alternative solution was proposed by the authors of [96]. The proposed algorithm is based on modification of a wavelet transform by replacement of wavelets on the boundaries by the so-called edge or interval wavelets. This allows for avoiding the problem of the boundary effect but additionally complicates the algorithm. In order to reduce the boundary effect and avoid the SDI algorithm complexification, Messina [97] proposed two different approaches. The first approach is based on isomorphism of a geometry of a tested structure, while the second one is based on self-minimization technique. These approaches allow eliminating the boundary effect even in the case of highly noised data $(\mathrm{SNR}=40 \mathrm{~dB})$. Montanari et al. [98] proposed an efficient padding method based on fitting polynomial functions, which has several advantages with respect to classical padding methods described above. Application of this method makes it possible to extend a signal without loss of continuity and if the damage exists in the boundary region it is not masked by the high-magnitude values of coefficients.

\section{Wavelet-Based Structural Damage Assessment (WavStructDamAs) Benchmark}

Due to the intensively developed wavelet-based algorithms for SDI problems over the last years, it was essential to develop a benchmark, which contains numerical data and data obtained from the vibration tests for testing of new algorithms. Naturally, the benchmark should contain the main wavelet tools for performing the analyses in order to be complimentary. The benchmark that meets these criteria has been developed and openly shared in the benchmark homepage: http://ipkm.polsl.pl/wavstructdamas. The benchmark contains 143 example problems of 1D, 2D, and 3D damaged composite structures, which are based on numerical and experimental data, and a possibility of uploading its own user data for performing the analyses using implemented wavelet transforms and supporting tools. The benchmark was implemented in Matlab environment as a GUI (Graphical User Interface) application. In order to make the benchmark available for a wide community, it was fully automated that no programming is necessary for using it. Moreover, it is distributed in the form of both Matlab routines and a stand-alone application, which does not require the Matlab environment. The benchmark includes example problems of various composite structures with various possible damage that may occur (cracks and notches, voids, delaminations, 
and impact damage). Most of them were described in separate papers listed on the home-page of the benchmark (the optimization studies were not included in the benchmark due to the very time-consuming algorithms-they are available upon request).

The benchmark is based on the Matlab environment with using the Matlab Wavelet Toolbox. Several wavelet transforms were implemented for various problems: for 1D problems, CWT, DWT, SWT, undecimated wavelet transform (UWT), dual-tree (complex) wavelet transform (DTWT), and FrDWT; for 2D problems, DWT, SWT, UWT, DTWT, DMWT, and QWT; for 3D problems, DWT and DTWT. Additional postprocessing tools cover the boundary effect removal functions of resulting coefficients regardless to their orientation (i.e., addition of coefficients after decomposition) using four algorithms. At every stage of processing, the data can be exported to the Matlab workspace (or to the text file in the case of stand-alone version), which does not limit the user to performing additional analyses or modify the existing routines.

3.1. Description of SDI Benchmark Problems. The implemented benchmark problems can be classified with respect to the problem dimension $(1 \mathrm{D}, 2 \mathrm{D}$, or $3 \mathrm{D})$ and the character of data (numerical or experimental). All of $1 \mathrm{D}$ and 2D numerical problems were modeled and computed in MSC Patran/Nastran or Marc/Mentat finite element software. The laminated composite structures were modeled as solids using 8-node hexagonal elements, where the parameters for layers were defined using appropriate modelers (definition of stiffness matrix for particular layers) or separately for every layer (in this case the ideal contact between the layers was assumed). The cracks and voids were modeled by excluding the appropriate elements from the model, while the delaminations were simulated by deactivation of a contact between layers in the region of interest. The detailed description including the materials properties as well as parameters of numerical analyses was described in several papers [44-46, $48,49,54,63]$.

The experimental studies were performed on laminated composite beams and plates, sandwich composite plates with a honeycomb core, and composite aircraft structures.

One-dimensional experimental problems in WavStructDamAs Benchmark consisted of two types: composite beams with single crack and multiple cracks. The tests were performed on laminated composite cantilever beams with dimensions of $250 \times 25 \mathrm{~mm}$ and a thickness of $5.28 \mathrm{~mm}$. The testing procedure was performed using scanning LDV Polytec PSV-400 and point LDV Polytec PDV-100 used as a reference on the length of $215 \mathrm{~mm}$ with a resolution of 44 (for single crack) and 39 (for multiple cracks) equidistant measurement points. The point LDV was focused on the surface of the clamping frame. The scanning LDV was connected with a vibrometer controller Polytec OFV-5000 with builtin velocity decoder and a PC. The excitation of specimens was performed by the electrodynamic shaker TIRA TV51120, which excited the specimen though the stinger by pseudorandom noise signal generated directly from the software dedicated to scanning LDV and amplified by the power amplifier TIRA BAA 500. The tested specimens with multiple cracks and the experimental setup with description are presented in Figure 1. From the obtained FRFs, the modal shapes of vibration were extracted and further subjected to wavelet analysis in order to detect and localize cracks. The details of experiment and results obtained using DWT-based algorithm were presented in [43].

A set of two-dimensional experimental problems in WavStructDamAs Benchmark contains the studies performed on laminated square composite plates with surface and impact damage, laminated circular composite plate with stiff inclusion, square sandwich composite plates with damaged core and impact damage, and aircraft composite structures with impact damage.

Laminated square composite plates with surface damage consisted of three types: through-the-width crack, spatial square damage, and multiple damage (sites with various dimensions and a small surface crack). The dimensions of plates (both with surface and impact damage) are as follows: spatial dimensions of $300 \times 300 \mathrm{~mm}$ and a thickness of $2.5 \mathrm{~mm}$. All of the surface damage was simulated using a numerical milling machine. The depth of simulated damage equaled $0.5 \mathrm{~mm}$ ( $20 \%$ of total thickness of the tested plates). The plates were clamped in a steel frame in such a way that the internal square of the frame had the edge-length of $250 \mathrm{~mm}$. The testing procedure used the same experimental configuration (two LDVs with supporting equipment) as in the case of composite beams described above. The scanning procedure was performed on the surface area of plates of 250 $\times 250 \mathrm{~mm}$ and setting the net of $64 \times 64$ equidistant measurement points. The schemes of simulated damage with specific dimensions and experimental setup are presented in Figure 2.

The damage identification studies performed on these plates using various approaches were described in $[44,54,63]$.

Example problems of impact damage identification in laminated composite plates were performed on the same type of plates and using the same experimental setup as for the plates with surface damage. The impact damage was simulated on the own-designed test rig using various values of impact energy and various impactors. The test rig and impactors are presented in Figure 3.

The exemplary FRF with selected modes for analysis is visualized in Figure 4. Damage identification studies performed on these plates were described in [50].

An example problem concerned with 12-layer laminated composite circular plate was based on experimental tests of a plate with diameter of $500 \mathrm{~mm}$ and a thickness of $2.1 \mathrm{~mm}$. For the testing purposes the plate was mounted directly on the shaker. For the mounting purpose a circular hole was cut in the center of a plate with a diameter of $60 \mathrm{~mm}$. During the lamination process the sector-shaped PTFE (polytetrafluoroethylene) tape was inserted between sixth and seventh layers. The inclusion has the radial dimensions same as the radial dimensions of a plate and the angular dimension of $20^{\circ}$. The plate was clamped in its center using a steel disc with a diameter of $78 \mathrm{~mm}$. Using the same test equipment as previously, the modal analysis was performed using a net of $64 \times 64$ measurement points (in radial and angular directions). 

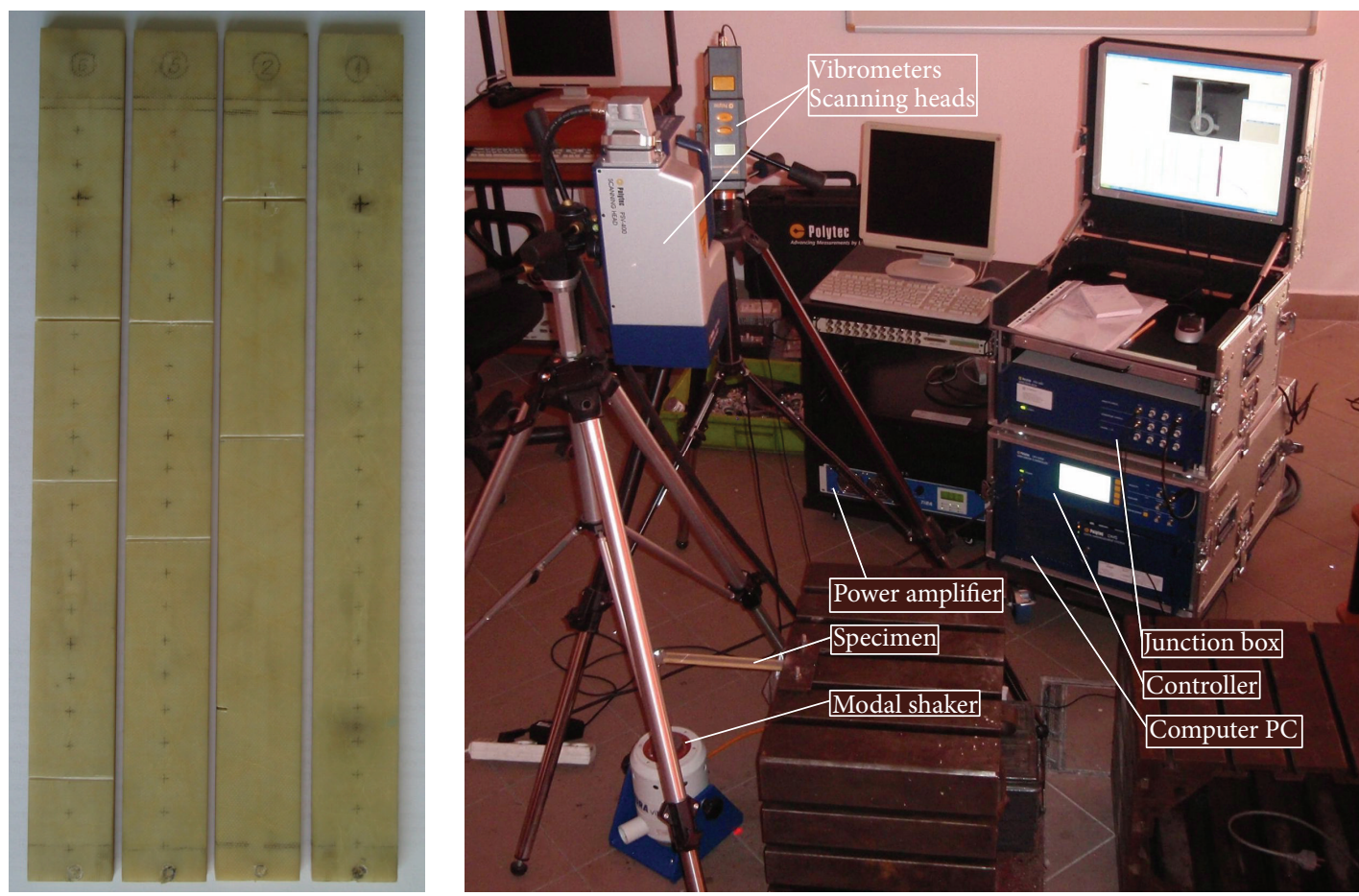

FIGURE 1: Specimens with multiple cracks and experimental setup.
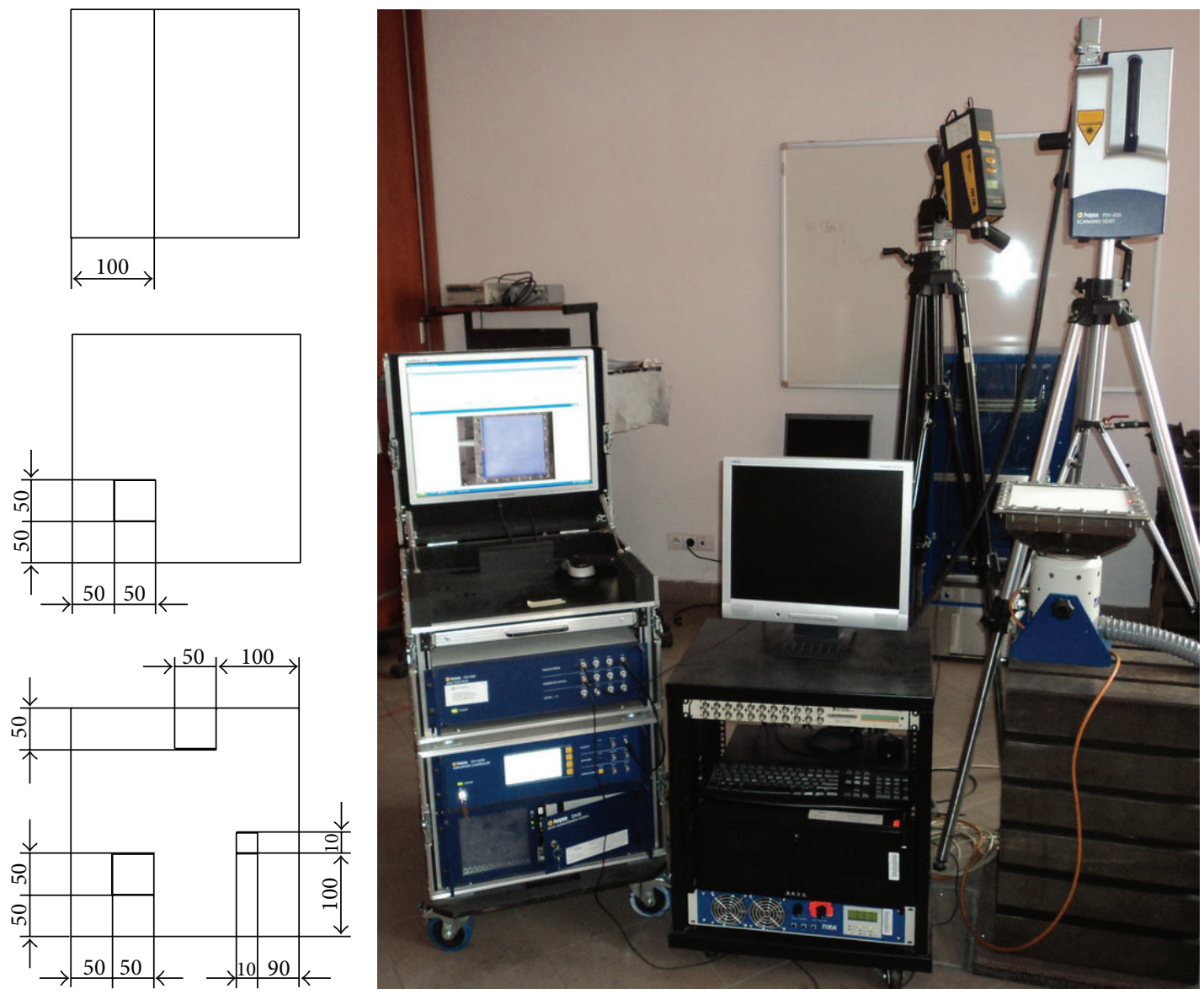

FIGURE 2: Schemes of surface damage of tested plates and experimental setup. 

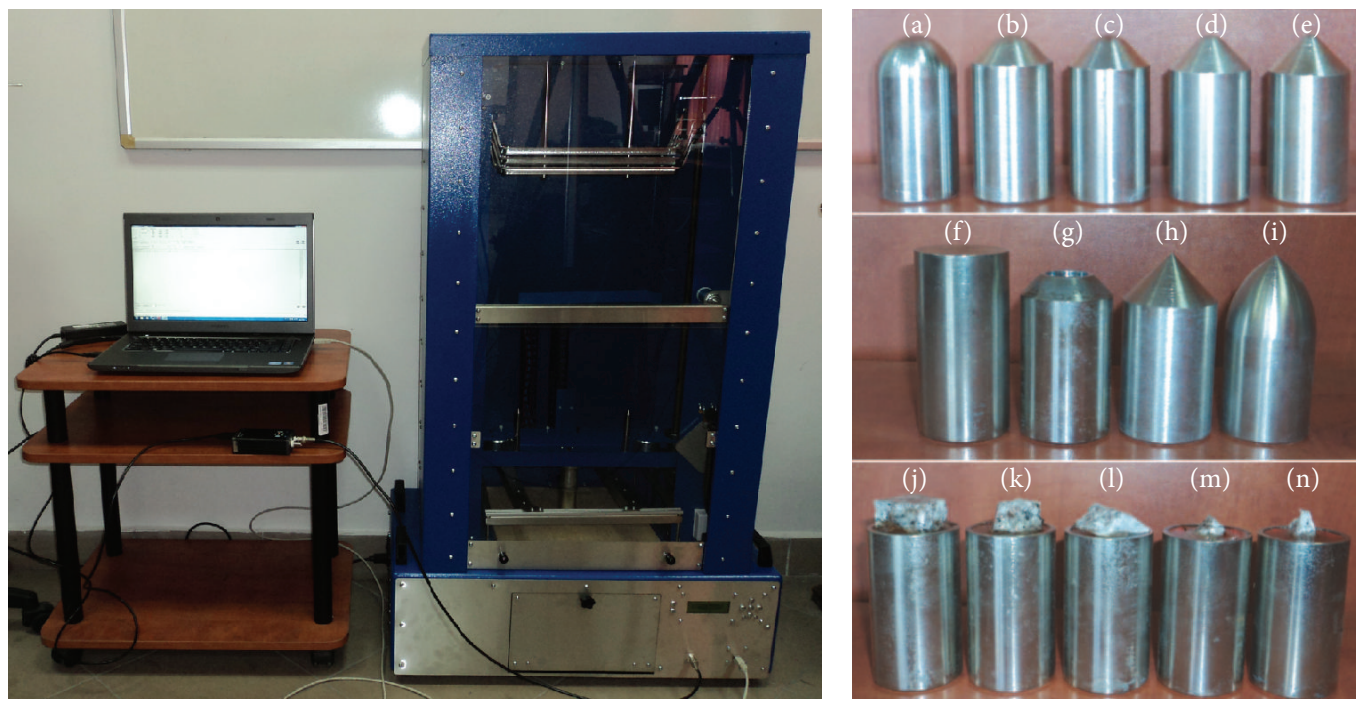

FiguRE 3: Impactors with rounded ends: (a) R17, (b) R14, (c) R11, (d) R8, and (e) R5 mm; impactors: (f) with a flat end ø34 mm, (g) with a flat end $ø 19 \mathrm{~mm}$, (h) with the conic end, and (i) with the arched end; impactors with immersed stones: (j) with flat impact surface, (k) with rough impact surface, (l) edge-ended, (m) vertex-ended, and (n) angle-oriented vertex-ended.

The experimental setup for these tests is shown in Figure 5. A detailed description of identification of stiff inclusion in this plate is available in [49].

Sandwich plates of spatial dimensions of $300 \times 300 \mathrm{~mm}$ and a thickness of $4.1 \mathrm{~mm}$ used for example problems implemented in WavStructDamAs Benchmark consisted of a core with a thickness of $3 \mathrm{~mm}$ manufactured from the aramid paper saturated by phenolic resin with hexagonal cell configuration. The face sheets were manufactured in the form of glass-fiber reinforced laminate made of plain weave glass fabric. The damage of a core was introduced during the manufacturing process before laying-up with face sheets. Four cases were considered: through-the-width crack of a core, partial local lack of a core with irregular boundary placed in the center of a plate, full local lack of a core with irregular boundary placed in the center of a plate, and the delamination between a core and upper face sheet of a square shape with dimensions of $60 \times 60 \mathrm{~mm}$ placed in the center of a plate. The impact damage was introduced using the impact test machine (see Figure 3) with various impactors and various values of impact energy. The tests were performed under similar conditions as in the case of laminated composite plates; that is, the plates were clamped in the steel frame and the modal analysis was performed using two LDVs. The detailed description and results obtained using FrDWT-based algorithm were presented in [55].

Three example problems of impact damage identification were implemented in the benchmark. The first structure was manufactured in the form of glass fiber-reinforced polymeric (GFRP) composite with dimensions of $205 \times 255 \mathrm{~mm}$. Three barely visible impact damage (BVID) with impact energy of 3 , 6 , and $9 \mathrm{~J}$, respectively, were introduced in the structure. The plate was clamped on two opposite edges in a steel frame and scanned using a testing configuration of two LDVs described above on the surface area of $205 \times 245 \mathrm{~mm}$ on the net of
$59 \times 71$ equidistant points. The second tested structure was made in the form of hybrid Al-GFRP-Al composite with dimensions of $200 \times 255 \mathrm{~mm}$. It was scanned in the same way on the area of $190 \times 250 \mathrm{~mm}$ with a resolution of $73 \times$ 51 measurement points. The third tested structure was an extraction from a vertical stabilizer of a military aircraft reinforced by stiffeners and rivet holes made of carbon fiberreinforced polymer with spatial dimensions of $270 \times 300 \mathrm{~mm}$. The scanning procedure was performed on the area of $260 \times$ $300 \mathrm{~mm}$ on the net of $73 \times 65$ measurement points. The aircraft structures were manufactured in the Air Force Institute of Technology in Warsaw, Poland. The images of these structures are presented in Figure 6. A detailed description of these studies as well as comparison of the obtained results with results obtained by other NDT methods can be found in [99].

The benchmark includes two additional example problems in 3D. In these cases the experimental data were collected based on scanning of composite structures using computed tomography (CT) technique. One of the tested plates with dimensions of $300 \times 150 \mathrm{~mm}$ and a thickness of $5 \mathrm{~mm}$ was manufactured as CFRP structure, while the second plate with dimensions of $800 \times 150 \mathrm{~mm}$ and a thickness of $2 \mathrm{~mm}$ was prepared in the form of multilayer weft knitted fabrics in the Institute of Lightweight Engineering and Polymer Technology (ILK), TU Dresden, Germany (Figure 7). The manufacturing details and material properties for these plates can be found in [100].

In both plates, the circular holes of diameter of $30 \mathrm{~mm}$ in the middle of specimens were cut using water-jet cutting method on the Trumpf Trumatic WS 2500 cutting system. During this process, the delaminated areas occurred around the holes. During the tomographic tests 3D data arrays of 452 $\times 504 \times 83$ voxels and $434 \times 507 \times 100$ voxels, respectively, were obtained. The analysis using 3D DWT was performed on the collected data in order to identify and classify damage 


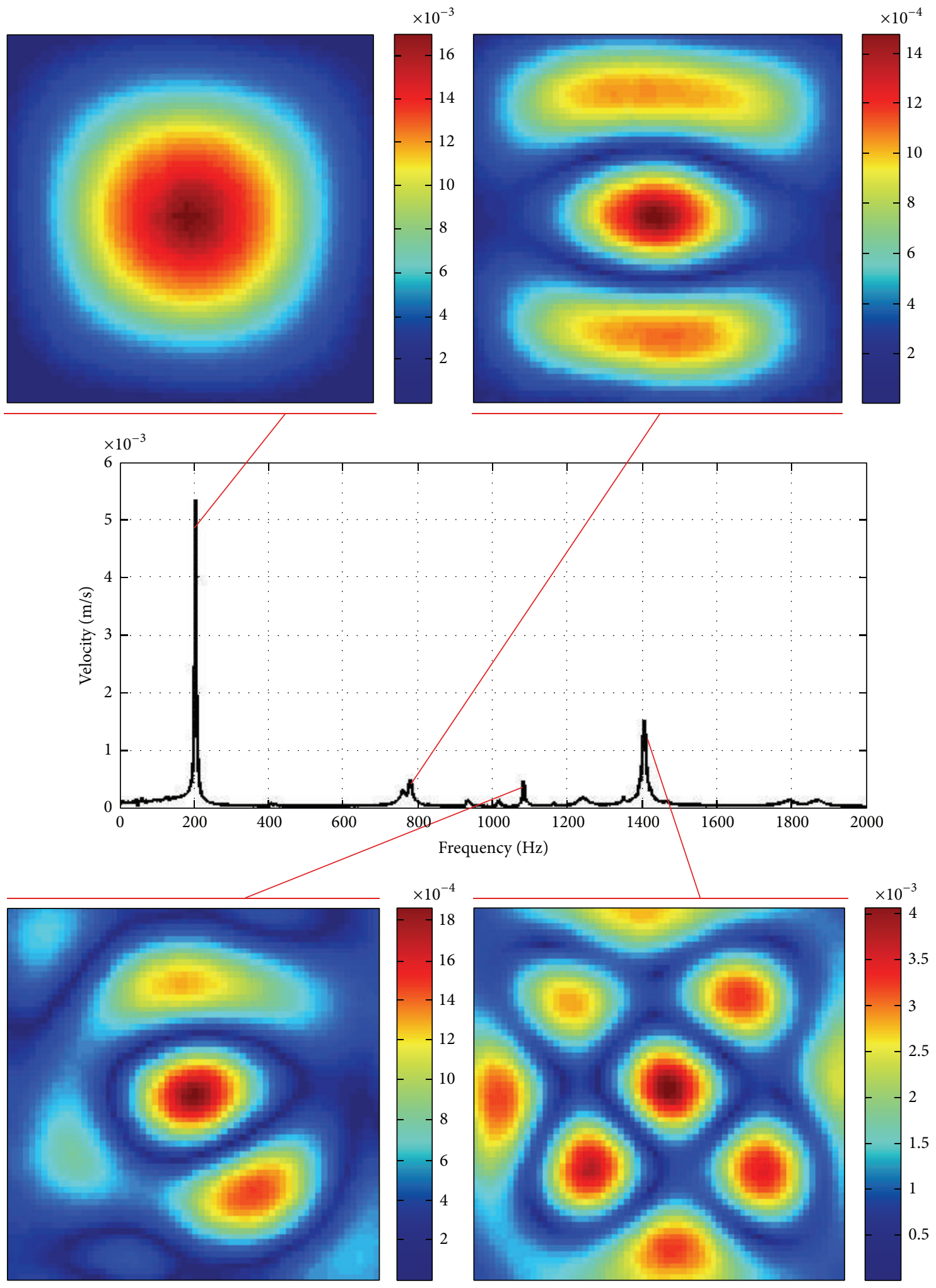

FIGURE 4: Exemplary FRF and selected modal shapes for the analysis. 


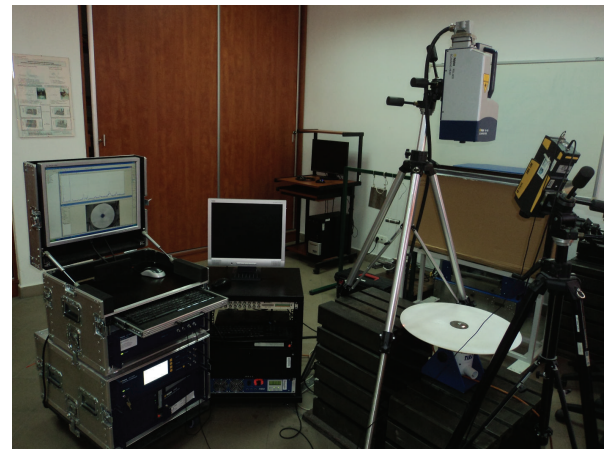

FIGURE 5: Experimental setup for identification of stiff inclusion in composite circular plate.
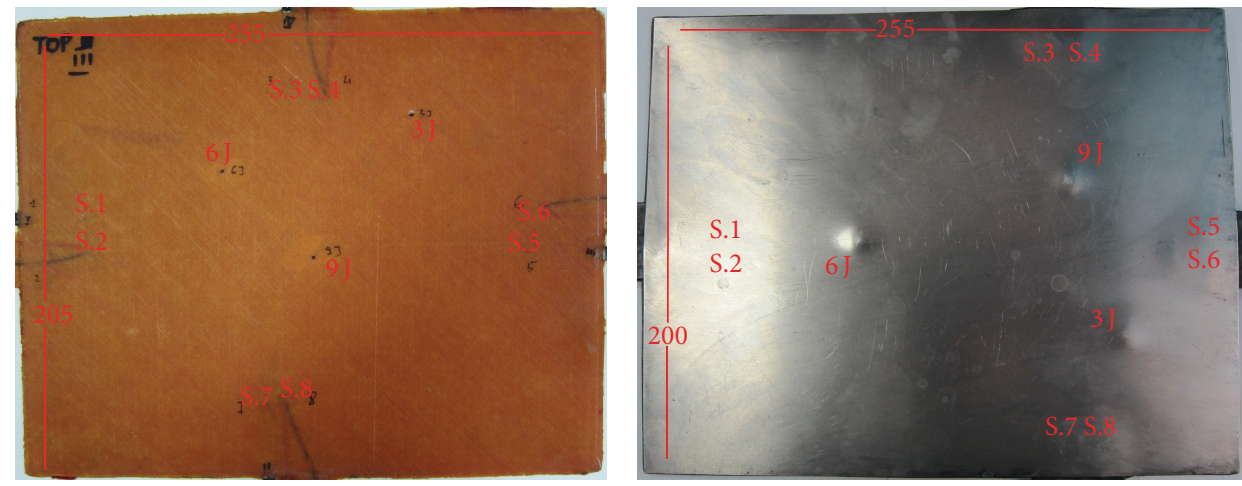

(a)
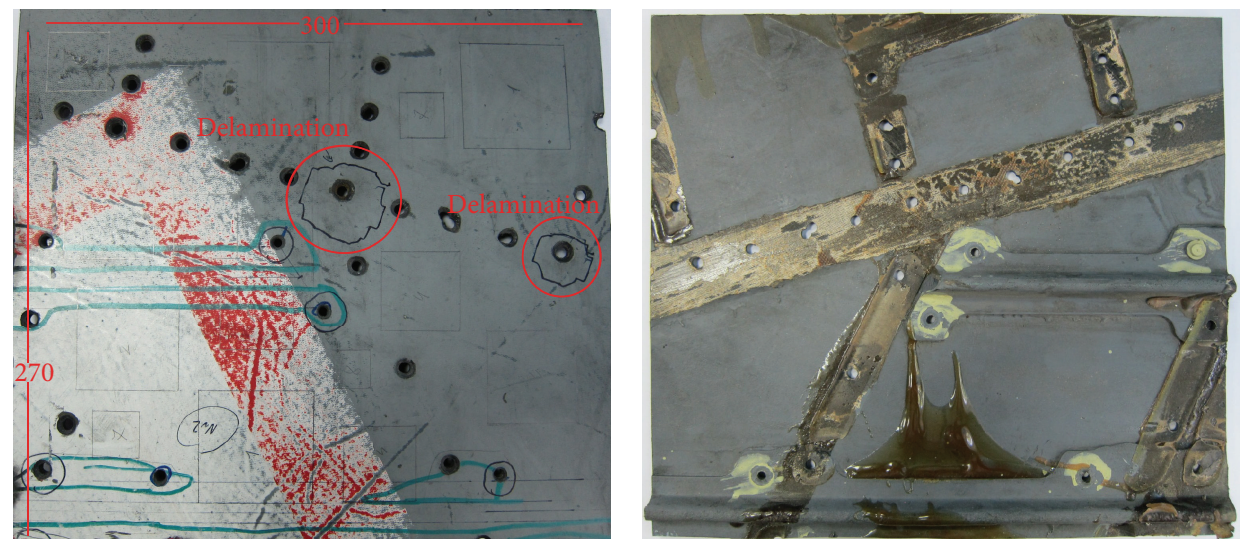

(b)

FIgURE 6: GFRP, Al-GFRP-Al, and CFRP extraction from the vertical stabilizer of the aircraft (a and b).
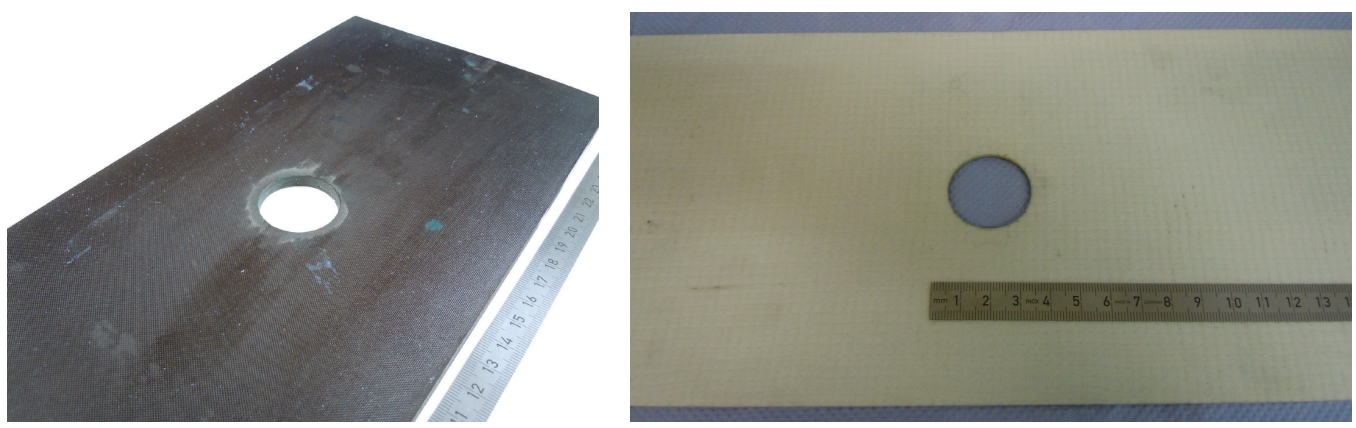

FIGURE 7: CFRP and multilayer weft knitted fabrics composite plates subjected to CT testing. 


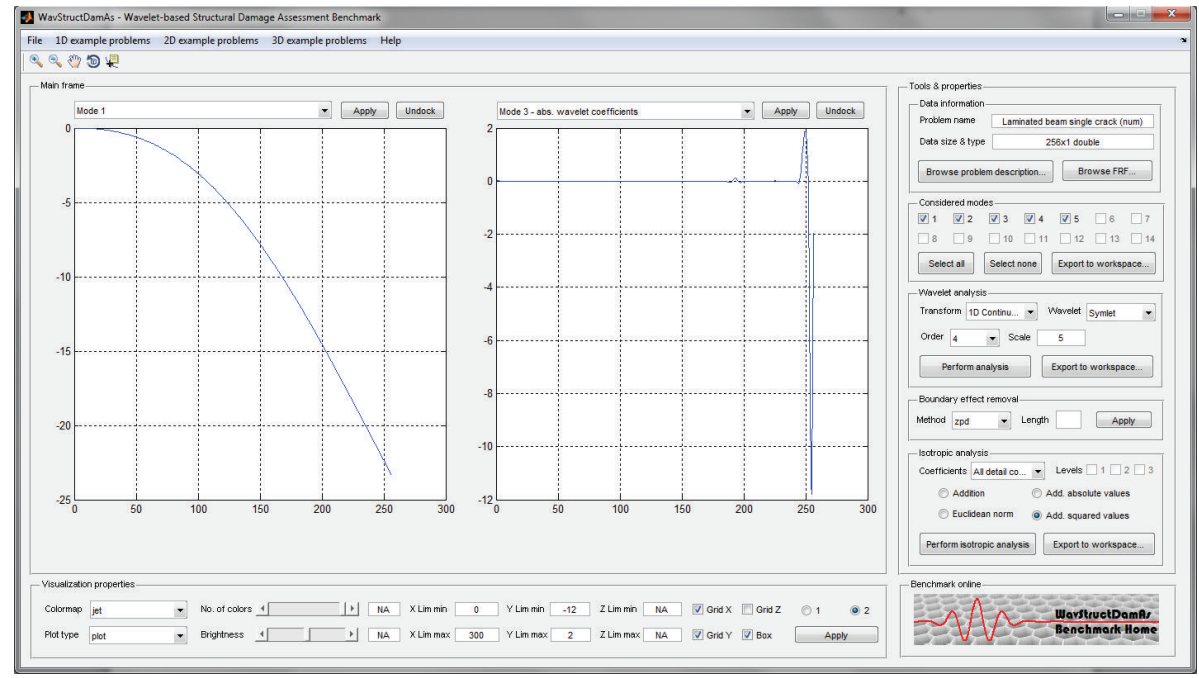

FIGURE 8: A benchmark window with results of laminated composite beam with a single crack example problem obtained using CWT-based approach.

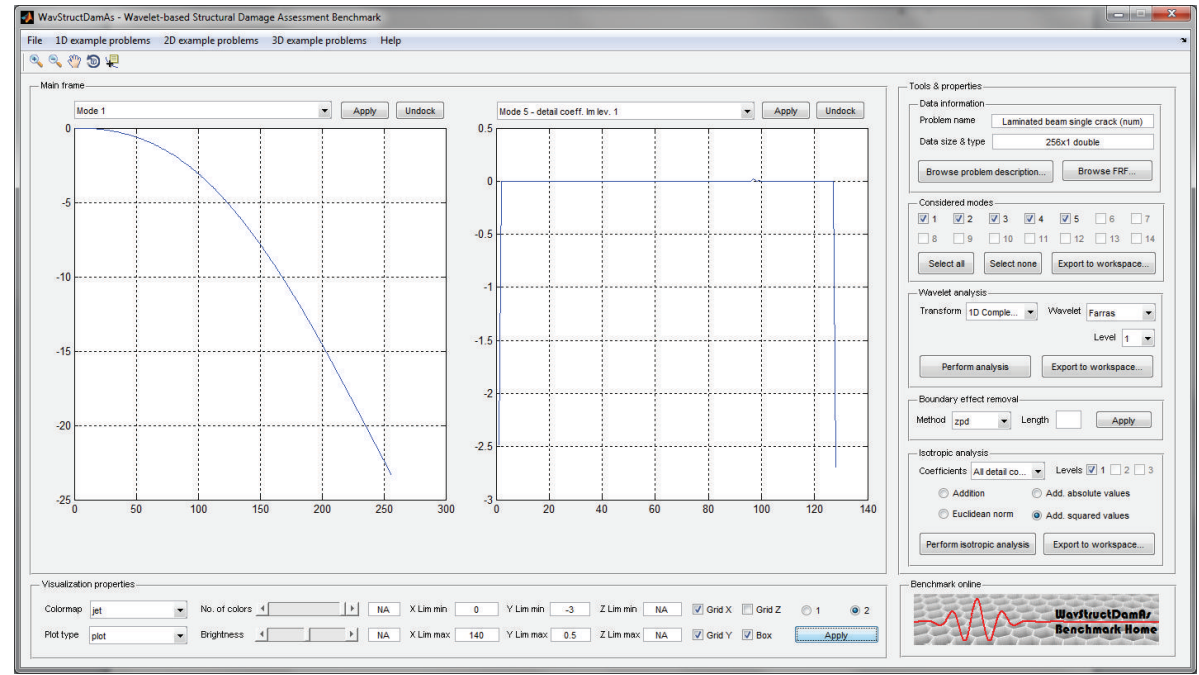

FIGURE 9: A benchmark window with results of laminated composite beam with a single crack example problem obtained using DTWT-based approach.

types in the tested structures. The detailed description of damage location and results of the analysis can be found in [101].

The problems described above implemented in the WavStructDamAs Benchmark can be analyzed using available wavelet-based algorithms in various combinations. In order to demonstrate the benchmark performance several exemplary analyses are presented in the next section.

\subsection{Exemplary Analyses Using WavStructDamAs Benchmark.} The first demonstration is performed on the example problem of laminated composite beam with a single crack. The data obtained from numerical model consisted of 5 modal shapes, each of 256 measurement points. After selection of this example problem the modal shapes become available and the first modal shape is visualized (see Figure 8, left frame).
From the problem description window (available in data information panel) one can determine the dimensions of an investigated beam and the position of damage. Two analysis types were selected in this case: 1D continuous wavelet transform and $1 \mathrm{D}$ complex wavelet transform. In both cases the symlet of order 4 was applied for the analyses. The CWTbased analysis was performed with a scale parameter of 5 . The DTWT-based analysis was performed as single-level decomposition. The results of the analysis are available in the right frame (Figure 8). The benchmark windows with selected results for each analysis are presented in Figures 8 and 9 , respectively.

One can observe that the damage is detectable (see the results frames in Figures 8 and 9); however the resulting coefficients are highly biased by the boundary effect. In order to remove this effect the boundary effect removal tool was 


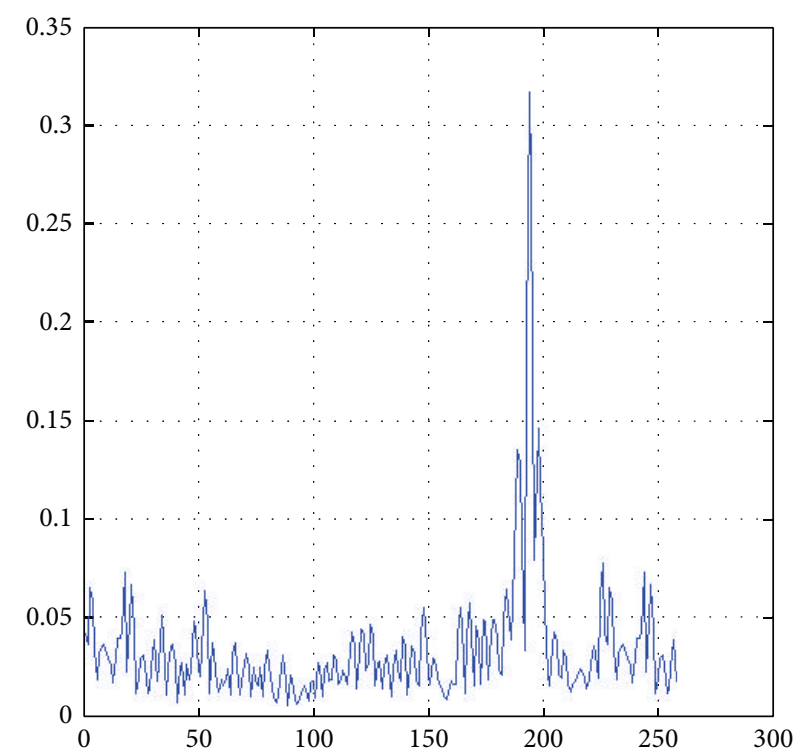

(a)

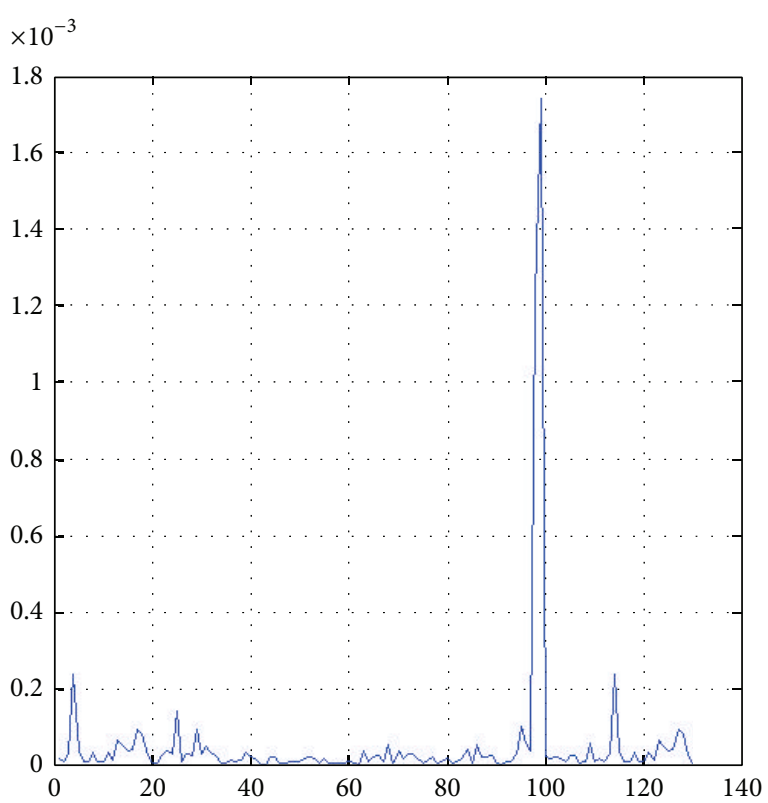

(b)

FIGURE 10: Benchmark results for SDI using isotropic CWT-based (a) and DTWT-based (b) algorithms with boundary effect removal.

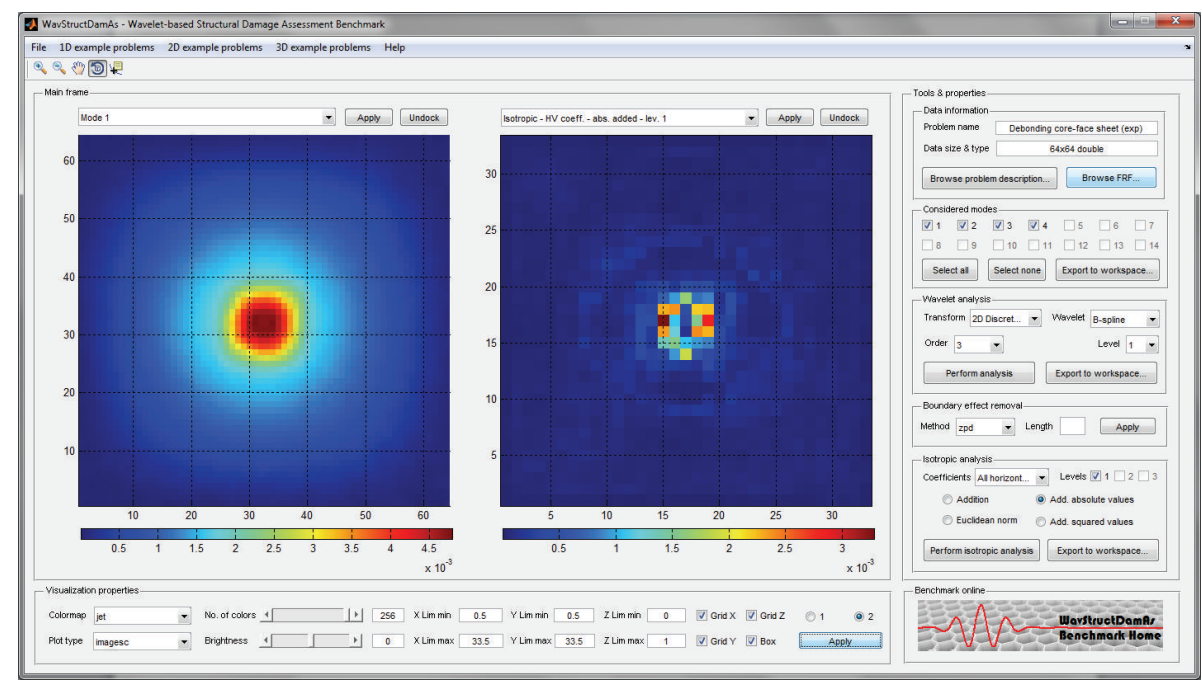

FIGURE 11: The benchmark results for SDI in a sandwich plate using isotropic DWT-based algorithm.

applied: in the case of CWT-based analysis the extension length was equaled to 16 points, while for DTWT-based analysis the extension length was equaled to 10 points. In both cases the periodization technique was applied for the signal extension. Analyzing obtained results, one can observe that the $3 \mathrm{rd}$ and 5 th modal shapes are sensitive to the damage. In order to visualize the results of SDI for the discussed example problem, the isotropic analysis was performed, where all obtained detail coefficients were squared and added up. Obtained plots with detectable damage positions were exported to separate windows and are presented in Figure 10.

The next demonstration of benchmark performance is based on example problem of debonding between a core and a face sheet in a sandwich plate. Four modal shapes are available for this example problem. The analysis was performed using 2D DWT-based algorithm with the Bspline wavelet of order 3 as single-level decomposition. After performing the analysis the absolute values of obtained horizontal and vertical coefficients were added up. A window with obtained results is presented in Figure 11. The squareshaped debonding can be easily detected and localized.

The benchmark allows for analysis of circular-shaped structures. In the next demonstrated example problem the circular laminated composite with PTFE sector-shaped inclusion is analyzed. The analysis was performed using $2 \mathrm{D}$ FrDWT-based algorithm using fractional B-spline wavelets 


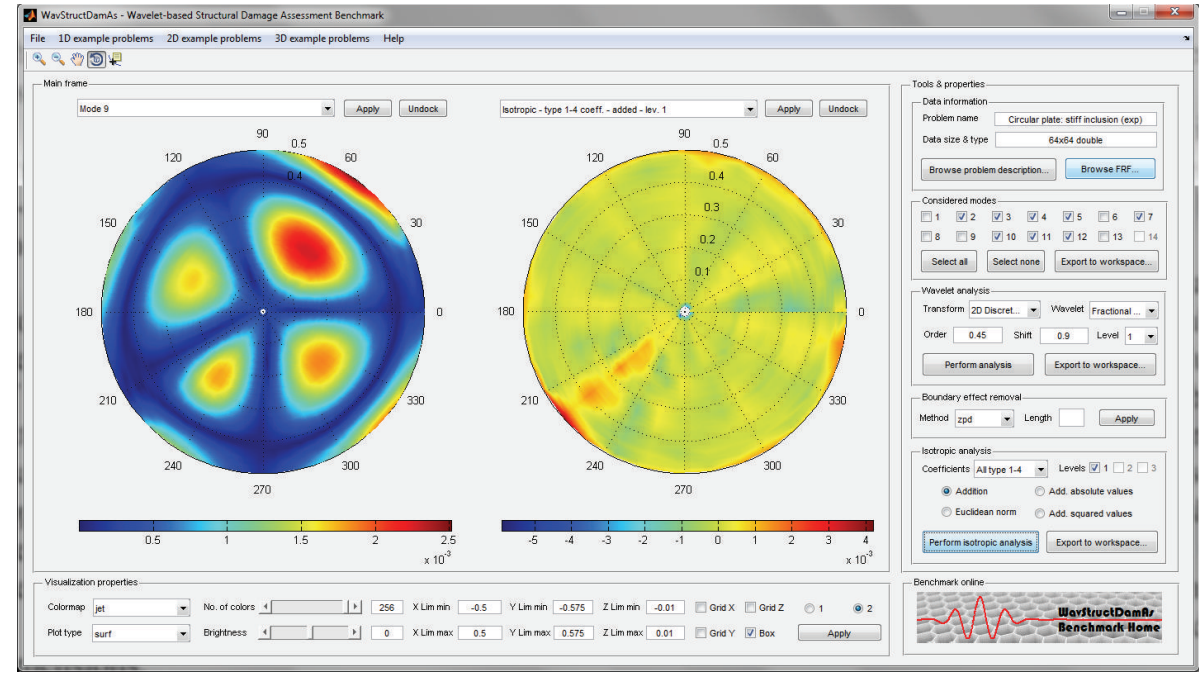

FIGURE 12: The benchmark results for SDI in a circular composite plate with stiff inclusion using isotropic FrDWT-based algorithm applied to selected modal shapes.

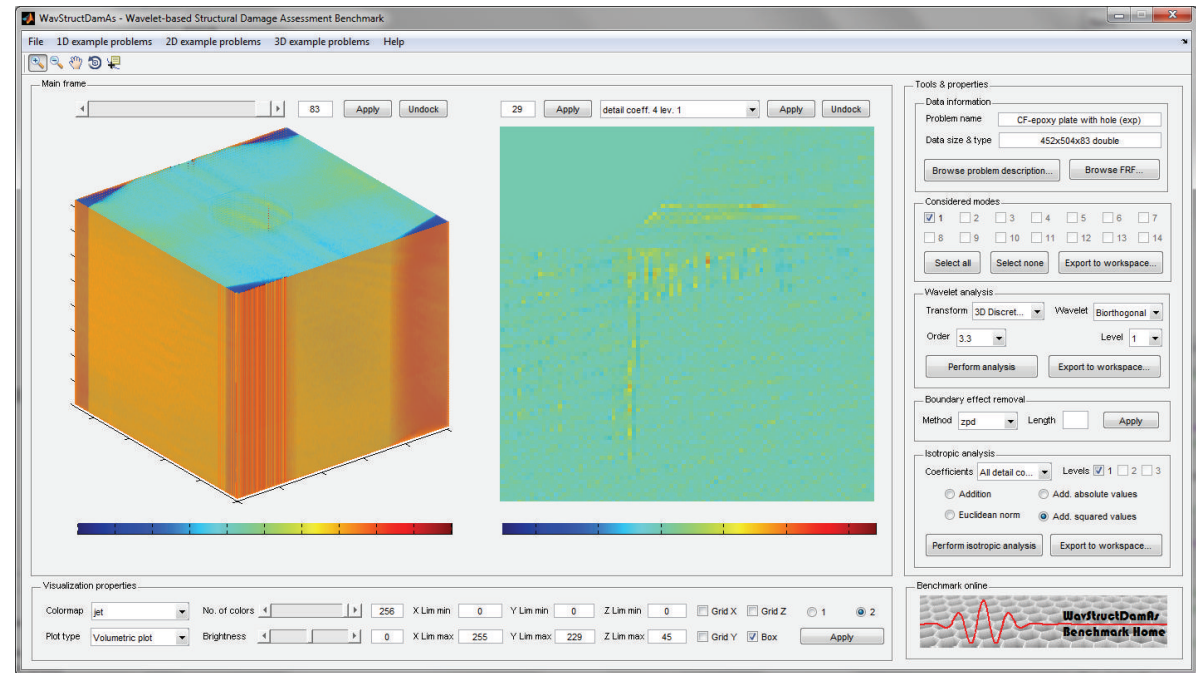

FIGURE 13: The benchmark results for SDI in CFRP plate with a hole and delaminations using 3D DWT-based algorithm.

of order 0.45 and a shift factor of 0.9 on selected modal shapes of a plate $(2-5,7$, and $10-12)$. Further, from the obtained coefficients the real and imaginary ones of types 1-4 were added up for the considered modal shapes. The sectorshaped PTFE inclusion is detected and localized properly (see Figure 12).

Finally, one of the 3D example problems is demonstrated. The analysis was performed on CT data of CFRP plate with a hole and delaminations using 3D DWT-based algorithm. A biorthogonal wavelet of order 3.3 is applied. The performed decomposition allows for detection of delamination directions in particular cross sections of a tested plate. In Figure 13 the exemplary results are shown. Obtaining the detail coefficients of type 4 (detail-detail-approximation) allowed detecting the boundaries of delaminated areas. The resulting set of coefficients presented in Figure 13 was firstly cross-sectioned in the 29th layer of $Z$-direction and then rotated to $X-Y$ view and magnified for improving the visualization of the detected damage.

The performance and capabilities of WavStructDamAs benchmark are not limited to the demonstrated cases and can be used for studying of both available example problems and its own problems, which can be uploaded by user, using available wavelet transforms and supporting tools for wavelet-based SDI.

\section{Conclusions}

The presented state-of-the-art review in the field of structural damage detection and identification using wavelet-based methods covers the presentation of results of the most important studies in this field as well as activity of the author and his team. Several important factors that influence the detectability and accuracy of localization of various types of 
damage were analyzed. The analysis includes the influence of measurement apparatus and resulting measurement noise, influence of applied wavelet transforms and wavelets and the current trends of improvement of existing wavelet-based methods applied for SDI, and the influence of damage location, sampling distance, and boundary effect on damage detection and localization with discussion on possible ways of elimination of unwanted effects and overall improvement of detectability and accuracy in SDI.

Based on the results of numerical and experimental studies performed by the author and his collaborators over the last five years, the WavStructDamAs Benchmark has been developed and implemented in Matlab environment. The developed benchmark has several advantages. First of all, it contains 143 example problems based on numerical and experimental data obtained from testing of real composite structures with a wide range of possible damage. Making this data openly available, the researchers in the SDI field can test the implemented algorithms or use their own ones to this data. Moreover, it is possible to upload its own user data; thus WavStructDamAs can be used as a toolbox. Finally, the benchmark has been developed as a fully GUI application; thus the coding for performing the analyses is not necessary. This creates a possibility of performing analyses easily and quickly and additionally using the benchmark by unexperienced Matlab users. The benchmark is distributed as an open-source software (users are able to modify and/or use available code in their own application) and a standalone application, which does not require Matlab installed on user's system. The benchmark can be useful for researchers working in the area of nondestructive testing, structural damage assessment, and structural health monitoring as well as for students attending to the corresponding university courses.

\section{Conflict of Interests}

The author declares that there is no conflict of interests regarding the publication of this paper.

\section{Acknowledgments}

The research project was financed by the National Science Centre (Poland) granted according to the Decision no. DEC2011/03/N/ST8/06205. The author would like to thank all the contributors to the performed experimental studies. The author also would like to thank two anonymous reviewers for valuable comments and suggestions which allow improving significantly this paper.

\section{References}

[1] D. Montalvão, N. M. M. Maia, and A. M. R. Ribeiro, "A review of vibration-based structural health monitoring with special emphasis on composite materials," Shock and Vibration Digest, vol. 38, no. 4, pp. 295-324, 2006.

[2] A. K. Pandey, M. Biswas, and M. M. Samman, "Damage detection from changes in curvature mode shapes," Journal of Sound and Vibration, vol. 145, no. 2, pp. 321-332, 1991.
[3] Y. K. Ho and D. J. Ewins, "On the structural damage identification with mode shapes," in Proceedings of the European Conference on System Identification and Structural Health Monitoring, pp. 677-686, Madrid, Spain, 2000.

[4] C. S. Hamey, W. Lestari, P. Qiao, and G. Song, "Experimental damage identification of carbon/epoxy composite beams using curvature mode shapes," Structural Health Monitoring, vol. 3, no. 4, pp. 333-353, 2004.

[5] D. Wu and S. S. Law, "Damage localization in plate structures from uniform load surface curvature," Journal of Sound and Vibration, vol. 276, no. 1-2, pp. 227-244, 2004.

[6] Z. Ismail, H. Abdul Razak, and A. G. Abdul Rahman, "Determination of damage location in RC beams using mode shape derivatives," Engineering Structures, vol. 28, no. 11, pp. 15661573, 2006.

[7] J. F. Gauthier, T. M. Whalen, and J. Liu, "Experimental validation of the higher-order derivative discontinuity method for damage identification," Structural Control and Health Monitoring, vol. 15, no. 2, pp. 143-161, 2008.

[8] P. Moreno-García, J. V. Araújo dos Santos, and H. Lopes, "A new technique to optimize the use of mode shape derivatives to localize damage in laminated composite plates," Composite Structures, vol. 108, no. 1, pp. 548-554, 2013.

[9] M. S. Cao, M. Radzieński, W. Xu, and W. Ostachowicz, "Identification of multiple damage in beams based on robust curvature mode shapes," Mechanical Systems and Signal Processing, vol. 46, no. 2, pp. 468-480, 2014.

[10] C. P. Ratcliffe, "Damage detection using a modified laplacian operator on mode shape data," Journal of Sound and Vibration, vol. 204, no. 3, pp. 505-517, 1997.

[11] M. M. A. Wahab and G. D. De Roeck, "Damage detection in bridges using modal curvatures: application to a real damage scenario," Journal of Sound and Vibration, vol. 226, no. 2, pp. 217-235, 1999.

[12] M. Chandrashekhar and R. Ganguli, "Structural damage detection using modal curvature and fuzzy logic," Structural Health Monitoring, vol. 8, no. 4, pp. 267-282, 2009.

[13] E. Sazonov and P. Klinkhachorn, "Optimal spatial sampling interval for damage detection by curvature or strain energy mode shapes," Journal of Sound and Vibration, vol. 285, no. 4-5, pp. 783-801, 2005.

[14] M. K. Yoon, D. Heider, J. W. Gillespie Jr., C. P. Ratcliffe, and R. M. Crane, "Local damage detection using the two-dimensional gapped smoothing method," Journal of Sound and Vibration, vol. 279, no. 1-2, pp. 119-139, 2005.

[15] J.-H. Chou and J. Ghaboussi, "Genetic algorithm in structural damage detection," Computers \& Structures, vol. 79, no. 14, pp. 1335-1353, 2001.

[16] J. N. Yang, Y. Lei, S. Lin, and N. Huang, "Hilbert-Huang based approach for structural damage detection," Journal of Engineering Mechanics, vol. 130, no. 1, pp. 85-95, 2004.

[17] M. Imregun and W. J. Visser, "A review of model updating techniques," Shock \& Vibration Digest, vol. 23, no. 1, pp. 9-20, 1991.

[18] J. E. Mottershead and M. I. Friswell, "Model updating in structural dynamics: a survey," Journal of Sound and Vibration, vol. 167, no. 2, pp. 347-375, 1993.

[19] S. V. Modak, T. K. Kundra, and B. C. Nakra, "Comparative study of model updating methods using simulated experimental data," Computers and Structures, vol. 80, no. 5-6, pp. 437-447, 2002. 
[20] H. H. Khodaparast, J. E. Mottershead, and M. I. Friswell, "Perturbation methods for the estimation of parameter variability in stochastic model updating," Mechanical Systems and Signal Processing, vol. 22, no. 8, pp. 1751-1773, 2008.

[21] W. Wang, J. E. Mottershead, and C. Mares, "Mode-shape recognition and finite element model updating using the Zernike moment descriptor," Mechanical Systems and Signal Processing, vol. 23, no. 7, pp. 2088-2112, 2009.

[22] W. Wang, J. E. Mottershead, C. M. Sebastian, and E. A. Patterson, "Shape features and finite element model updating from full-field strain data," International Journal of Solids and Structures, vol. 48, no. 11-12, pp. 1644-1657, 2011.

[23] S. Mallat, A Wavelet Tour of Signal Processing: The Sparse Way, Academic Press, Burlington, Mass, USA, 2008.

[24] S. H. Mortazavi and S. M. Shahrtash, "Comparing denoising performance of DWT, WPT, SWT and DT-CWT for partial discharge signals," in Proceedings of the 43rd International Universities Power Engineering Conference (UPEC '08), pp. 1-6, Padova, Italy, September 2008.

[25] C. Surace and R. Ruotolo, "Crack detection of a beam using the wavelet transform," in Proceedings of the 12th International Modal Analysis Conference, pp. 1141-1147, Honolulu, Hawaii, USA, 1994.

[26] K. M. Liew and Q. Wang, "Application of wavelet theory for crack identification in structures," Journal of Engineering Mechanics, vol. 124, no. 2, pp. 152-157, 1998.

[27] Q. Wang and X. Deng, "Damage detection with spatial wavelets," International Journal of Solids and Structures, vol. 36, no. 23, pp. 3443-3468, 1999.

[28] C.-C. Chang and L.-W. Chen, "Vibration damage detection of a Timoshenko beam by spatial wavelet based approach," Applied Acoustics, vol. 64, no. 12, pp. 1217-1240, 2003.

[29] C.-C. Chang and L.-W. Chen, "Damage detection of a rectangular plate by spatial wavelet based approach," Applied Acoustics, vol. 65 , no. 8, pp. 819-832, 2004.

[30] C.-C. Chang and L.-W. Chen, "Detection of the location and size of cracks in the multiple cracked beam by spatial wavelet based approach," Mechanical Systems and Signal Processing, vol. 19, no. 1, pp. 139-155, 2005.

[31] J.-C. Hong, Y. Y. Kim, H. C. Lee, and Y. W. Lee, "Damage detection using the Lipschitz exponent estimated by the wavelet transform: applications to vibration modes of a beam," International Journal of Solids and Structures, vol. 39, no. 7, pp. 18031816, 2002.

[32] E. Douka, S. Loutridis, and A. Trochidis, "Crack identification in beams using wavelet analysis," International Journal of Solids and Structures, vol. 40, no. 13-14, pp. 3557-3569, 2003.

[33] E. Douka, S. Loutridis, and A. Trochidis, "Crack identification in plates using wavelet analysis," Journal of Sound and Vibration, vol. 270, no. 1-2, pp. 279-295, 2004.

[34] S. Loutridis, E. Douka, L. J. Hadjileontiadis, and A. Trochidis, "A two-dimensional wavelet transform for detection of cracks in plates," Engineering Structures, vol. 27, no. 9, pp. 1327-1338, 2005.

[35] A. Gentile and A. Messina, "On the continuous wavelet transforms applied to discrete vibrational data for detecting open cracks in damaged beams," International Journal of Solids and Structures, vol. 40, no. 2, pp. 295-315, 2003.

[36] M. Rucka and K. Wilde, "Damage location in beam and plate structures by wavelet analysis of experimentally determined mode shapes," Key Engineering Materials, vol. 293-294, pp. 313$320,2005$.
[37] M. Rucka and K. Wilde, "Crack identification using wavelets on experimental static deflection profiles," Engineering Structures, vol. 28, no. 2, pp. 279-288, 2006.

[38] M. Rucka and K. Wilde, "Application of continuous wavelet transform in vibration based damage detection method for beams and plates," Journal of Sound and Vibration, vol. 297, no. 3-5, pp. 536-550, 2006.

[39] W. Fan and P. Qiao, "A 2-D continuous wavelet transform of mode shape data for damage detection of plate structures," International Journal of Solids and Structures, vol. 46, no. 25-26, pp. 4379-4395, 2009.

[40] S. Zhong and S. O. Oyadiji, "Crack detection in simply supported beams using stationary wavelet transform of modal data," Structural Control and Health Monitoring, vol. 18, no. 2, pp. 169-190, 2011.

[41] D.-U. Sung, C.-G. Kim, and C.-S. Hong, "Monitoring of impact damages in composite laminates using wavelet transform," Composites Part B: Engineering, vol. 33, no. 1, pp. 35-43, 2002.

[42] H. Gökdağ and O. Kopmaz, "A new damage detection approach for beam-type structures based on the combination of continuous and discrete wavelet transforms," Journal of Sound and Vibration, vol. 324, no. 3-5, pp. 1158-1180, 2009.

[43] A. Katunin, "Identification of multiple cracks in composite beams using discrete wavelet transform," Scientific Problems of Machines Operation and Maintenance, vol. 45, no. 2, pp. 41-52, 2010.

[44] A. Katunin, "Damage identification in composite plates using two-dimensional B-spline wavelets," Mechanical Systems and Signal Processing, vol. 25, no. 8, pp. 3153-3167, 2011.

[45] A. Katunin and F. Holewik, "Crack identification in composite elements with non-linear geometry using spatial wavelet transform," Archives of Civil and Mechanical Engineering, vol. 13, no. 3, pp. 287-296, 2013.

[46] A. Katunin, "Vibration-based damage identification in composite circular plates using polar discrete wavelet transform," Journal of Vibroengineering, vol. 15, no. 1, pp. 355-363, 2013.

[47] A. Bagheri, G. G. Amiri, and S. A. S. Razzaghi, "Vibrationbased damage identification of plate structures via curvelet transform," Journal of Sound and Vibration, vol. 327, no. 3-5, pp. 593-603, 2009.

[48] A. Katunin, "Spatial damage identification in composite plates using multiwavelets," Journal of Applied Mathematics and Computational Mechanics, vol. 12, no. 3, pp. 69-78, 2013.

[49] A. Katunin, "Identification of stiff inclusion in circular composite plate based on quaternion wavelet analysis of modal shapes," Journal of Vibroengineering, vol. 16, no. 5, pp. 2545-2551, 2014.

[50] A. Katunin, "Stone impact damage identification in composite plates using modal data and quincunx wavelet analysis," Archives of Civil and Mechanical Engineering, vol. 15, no. 1, pp. 251-261, 2014.

[51] M. Unser and T. Blu, "Fractional splines and wavelets," SIAM Review, vol. 42, no. 1, pp. 43-67, 2000.

[52] A. Katunin, "Crack identification in composite beam using causal B-spline wavelets of fractional order," Modeling in Engineering, vol. 15, no. 46, pp. 57-63, 2013.

[53] K. N. Chaudhury and M. Unser, "Construction of Hilbert transform pairs of wavelet bases and Gabor-like transforms," IEEE Transactions on Signal Processing, vol. 57, no. 9, pp. 34113425, 2009.

[54] A. Katunin and P. Przystałka, "Damage assessment in composite plates using fractional wavelet transform of modal shapes with 
optimized selection of spatial wavelets," Engineering Applications of Artificial Intelligence, vol. 30, pp. 73-85, 2014.

[55] A. Katunin, "Vibration-based spatial damage identification in honeycomb-core sandwich composite structures using wavelet analysis," Composite Structures, vol. 118, pp. 385-391, 2014.

[56] A. Katunin, "Damage assessment in composite structures using modal analysis and 2D undecimated wavelet transform," Journal of Vibroengineering, vol. 16, no. 8, pp. 3939-3950, 2014.

[57] L. H. Yam, Y. J. Yan, and J. S. Jiang, "Vibration-based damage detection for composite structures using wavelet transform and neural network identification," Composite Structures, vol. 60, no. 4, pp. 403-412, 2003.

[58] M. Rucka and K. Wilde, "Neuro-wavelet damage detection technique in beam, plate and shell structures with experimental validation," Journal of Theoretical and Applied Mechanics, vol. 48, no. 3, pp. 579-604, 2010.

[59] H. Hein and L. Feklistova, "Computationally efficient delamination detection in composite beams using Haar wavelets," Mechanical Systems and Signal Processing, vol. 25, no. 6, pp. 2257-2270, 2011.

[60] J. Morlier, F. Bos, and P. Castéra, "Diagnosis of a portal frame using advanced signal processing of laser vibrometer data," Journal of Sound and Vibration, vol. 297, no. 1-2, pp. 420-431, 2006.

[61] M. Nguyen, X. Wang, Z. Su, and L. Ye, "Damage identification for composite structures with Bayesian network," in Proceedings of the Intelligent Sensors, Sensor Networks and Information Processing Conference, pp. 307-311, Melbourne, Australia, 2004.

[62] J. Xiang and M. Liang, "A two-step approach to multi-damage detection for plate structures," Engineering Fracture Mechanics, vol. 91, pp. 73-86, 2012.

[63] A. Katunin and P. Przystałka, "Detection and localization of delaminations in composite beams using fractional Bspline wavelets with optimized parameters," Eksploatacja $i$ Niezawodnosc-Maintenance and Reliability, vol. 15, no. 3, pp. 391-399, 2014.

[64] A. Katunin and P. Przystałka, "Meta-optimization method for wavelet-based damage identification in composite structures," in Proceedings of the Federated Conference on Computer Science and Information Systems, pp. 429-438, Warsaw, Poland, September 2014.

[65] C. Sujatha, Vibration and Acoustics: Measurement and Signal Analysis, Tata McGraw Hill Education Private, New Delhi, India, 2010.

[66] C. Smith, C. M. Akujuobi, P. Hamory, and K. Kloesel, "An approach to vibration analysis using wavelets in an application of aircraft health monitoring," Mechanical Systems and Signal Processing, vol. 21, no. 3, pp. 1255-1272, 2007.

[67] D. M. Siringoringo and Y. Fujino, "Experimental study of laser Doppler vibrometer and ambient vibration for vibration-based damage detection," Engineering Structures, vol. 28, no. 13, pp. 1803-1815, 2006.

[68] P. Qiao, W. Lestari, M. G. Shah, and J. Wang, "Dynamics-based damage detection of composite laminated beams using contact and noncontact measurement systems," Journal of Composite Materials, vol. 41, no. 10, pp. 1217-1252, 2007.

[69] M. Radzieński, M. Krawczuk, and M. Palacz, "Improvement of damage detection methods based on experimental modal parameters," Mechanical Systems and Signal Processing, vol. 25, no. 6, pp. 2169-2190, 2011.
[70] M. Rucka, "Damage detection in beams using wavelet transform on higher vibration modes," Journal of Theoretical and Applied Mechanics, vol. 49, no. 2, pp. 399-417, 2011.

[71] M. Cao, L. Cheng, Z. Su, and H. Xu, "A multi-scale pseudoforce model in wavelet domain for identification of damage in structural components," Mechanical Systems and Signal Processing, vol. 28, pp. 638-659, 2012.

[72] M. Cao, M. Radzieński, W. Xu, and W. Ostachowicz, "Identification of multiple damage in beams based on robust curvature mode shapes," Mechanical Systems and Signal Processing, vol. 46, no. 2, pp. 468-480, 2014.

[73] W. Xu, M. Cao, M. Radzieński et al., "Detecting multiple smallsized damage in beam-type structures by Teager energy of modal curvature shape," Journal of Vibroengineering, vol. 17, no. 1, pp. 275-286, 2015.

[74] W. Xu, M. Cao, W. Ostachowicz, M. Radzieński, and N. Xia, "Two-dimensional curvature mode shape method based on wavelets and Teager energy for damage detection in plates," Journal of Sound and Vibration, vol. 347, pp. 266-278, 2015.

[75] J. Shang, Y. He, D. Liu, H. Zang, and W. Chen, "Laser Doppler vibrometer for real-time speech-signal acquirement," Chinese Optics Letters, vol. 7, no. 8, pp. 732-733, 2009.

[76] W. Ostachowicz, P. Kudela, P. Malinowski, and T. Wandowski, "Damage localisation in plate-like structures based on PZT sensors," Mechanical Systems and Signal Processing, vol. 23, no. 6, pp. 1805-1829, 2009.

[77] Z. Su, L. Ye, and X. Bu, "A damage identification technique for $\mathrm{CF} / \mathrm{EP}$ composite laminates using distributed piezoelectric transducers," Composite Structures, vol. 57, no. 1-4, pp. 465-471, 2002.

[78] L. Yu and V. Giurgiutiu, "In situ 2-D piezoelectric wafer active sensors arrays for guided wave damage detection," Ultrasonics, vol. 48, no. 2, pp. 117-134, 2008.

[79] Y. J. Yan and L. H. Yam, "Online detection of crack damage in composite plates using embedded piezoelectric actuators/sensors and wavelet analysis," Composite Structures, vol. 58, no. 1, pp. 29-38, 2002.

[80] P. Qiao, K. Lu, W. Lestari, and J. Wang, "Curvature mode shape-based damage detection in composite laminated plates," Composite Structures, vol. 80, no. 3, pp. 409-428, 2007.

[81] Y. Wang, S. Yuan, and L. Qiu, "Improved wavelet-based spatial filter of damage imaging method on composite structures," Chinese Journal of Aeronautics, vol. 24, no. 5, pp. 665-672, 2011.

[82] V. La Saponara, C. Brandli, L. Arronche, and W. Lestari, “Gabor wavelet transform contours for the detection of uniaxial tensile damage in woven fiberglass/epoxy composites," Mechanics Research Communications, vol. 62, pp. 138-145, 2014.

[83] W. J. Staszewski, "Intelligent signal processing for damage detection in composite materials," Composites Science and Technology, vol. 62, no. 7-8, pp. 941-950, 2002.

[84] Y. Huang, D. Meyer, and S. Nemat-Nasser, "Damage detection with spatially distributed 2D Continuous Wavelet Transform," Mechanics of Materials, vol. 41, no. 10, pp. 1096-1107, 2009.

[85] A. Panopoulou, T. Loutas, D. Roulias, S. Fransen, and V. Kostopoulos, "Dynamic fiber Bragg gratings based health monitoring system of composite aerospace structures," Acta Astronautica, vol. 69, no. 7-8, pp. 445-457, 2011.

[86] S. Lu, M. Jiang, Q. Sui, Y. Sai, and L. Jia, "Low velocity impact localization system of CFRP using fiber Bragg grating sensors," Optical Fiber Technology, vol. 21, pp. 13-19, 2015. 
[87] B. Li, Z. Li, J. Zhou, L. Ye, and E. Li, "Damage localization in composite lattice truss core sandwich structures based on vibration characteristics," Composite Structures, vol. 126, pp. 3451, 2015.

[88] S.-T. Quek, Q. Wang, L. Zhang, and K.-K. Ang, "Sensitivity analysis of crack detection in beams by wavelet technique," International Journal of Mechanical Sciences, vol. 43, no. 12, pp. 2899-2910, 2001.

[89] A. V. Ovanesova and L. E. Suárez, "Applications of wavelet transforms to damage detection in frame structures," Engineering Structures, vol. 26, no. 1, pp. 39-49, 2004.

[90] S. Zhong and S. O. Oyadiji, "Sampling interval sensitivity analysis for crack detection by stationary wavelet transform," Structural Control and Health Monitoring, vol. 20, no. 1, pp. 4569, 2013.

[91] M. Feilner, D. van de Ville, and M. Unser, "An orthogonal family of quincunx wavelets with continuously adjustable order," IEEE Transactions on Image Processing, vol. 14, no. 4, pp. 499-510, 2005.

[92] D. Van De Ville, T. Blu, and M. Unser, "Isotropic polyharmonic B-splines: scaling functions and wavelets," IEEE Transactions on Image Processing, vol. 14, no. 11, pp. 1798-1813, 2005.

[93] V. Strela, Multiwavelet theory and applications [Ph.D. thesis], Massachusetts Institute of Technology, Cambridge, Mass, USA, 1996.

[94] M. Solís, M. Algaba, and P. Galvín, “Continuous wavelet analysis of mode shapes differences for damage detection," Mechanical Systems and Signal Processing, vol. 40, no. 2, pp. 645-666, 2013.

[95] D. Černá, V. Finĕk, M. Gottfried et al., "Boundary artifact reduction in wavelet image compression," in Mezinárodní Konference Technical Computing Prague, Prague, Czech Republic, 2009.

[96] P. Shui and Z. Bao, "Interval interpolating wavelets with robust boundary filters," Science in China. Series E. Technological Sciences, vol. 43, no. 3, pp. 287-296, 2000.

[97] A. Messina, "Refinements of damage detection methods based on wavelet analysis of dynamical shapes," International Journal of Solids and Structures, vol. 45, no. 14-15, pp. 4068-4097, 2008.

[98] L. Montanari, B. Basu, A. Spagnoli, and B. M. Broderick, "A padding method to reduce edge effects for enhanced damage identification using wavelet analysis," Mechanical Systems and Signal Processing, vol. 52-53, pp. 264-277, 2015.

[99] A. Katunin, K. Dragan, and M. Dziendzikowski, "Damage identification in aircraft composite structures: a case study using various non-destructive testing techniques," Composite Structures, vol. 127, pp. 1-9, 2015.

[100] B. Grübner, W. Hufenbach, R. Gottwald, M. Lepper, and B. Zhou, "Experimental and numerical validation of an analytical calculation method for notched fibre-reinforced multilayered composites under bending and compressive loads," in Proceedings of the 19th International Conference on Composite Materials, Montreal, Canada, 2013.

[101] A. Katunin, M. Dańczak, and P. Kostka, "Automated identification and classification of internal defects in composite structures using computed tomography and 3D wavelet analysis," Archives of Civil and Mechanical Engineering, vol. 15, no. 2, pp. 436-448, 2015. 

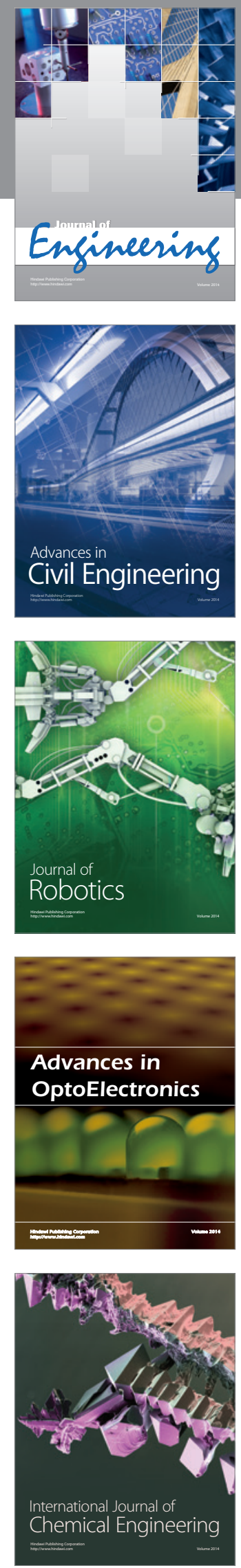

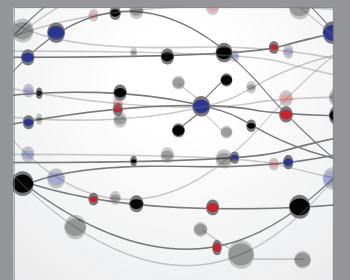

The Scientific World Journal
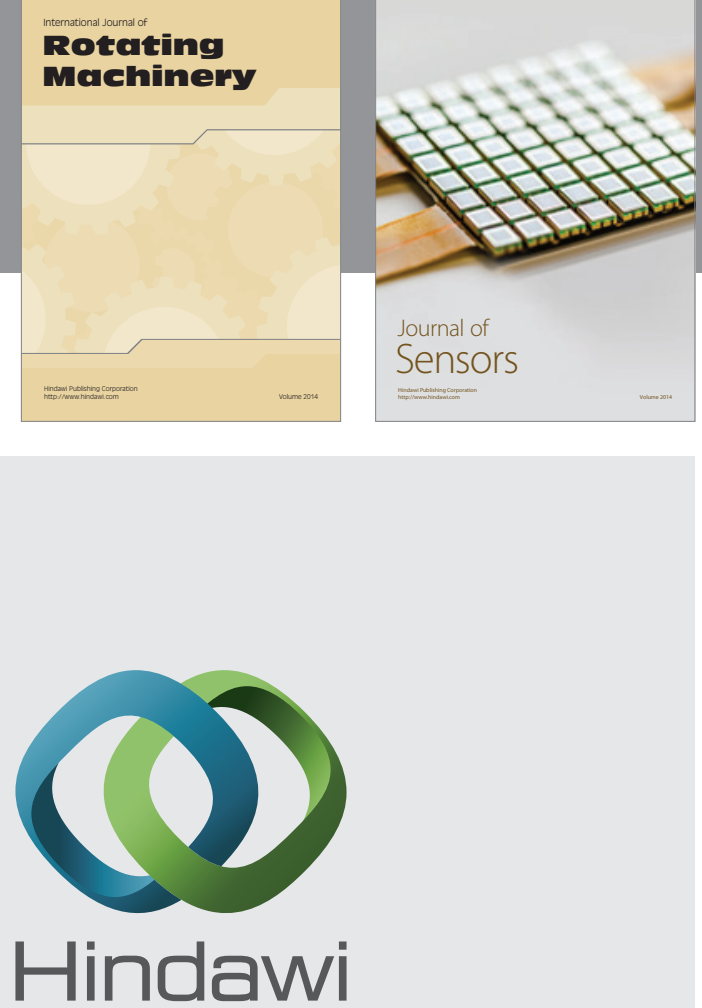

Submit your manuscripts at http://www.hindawi.com
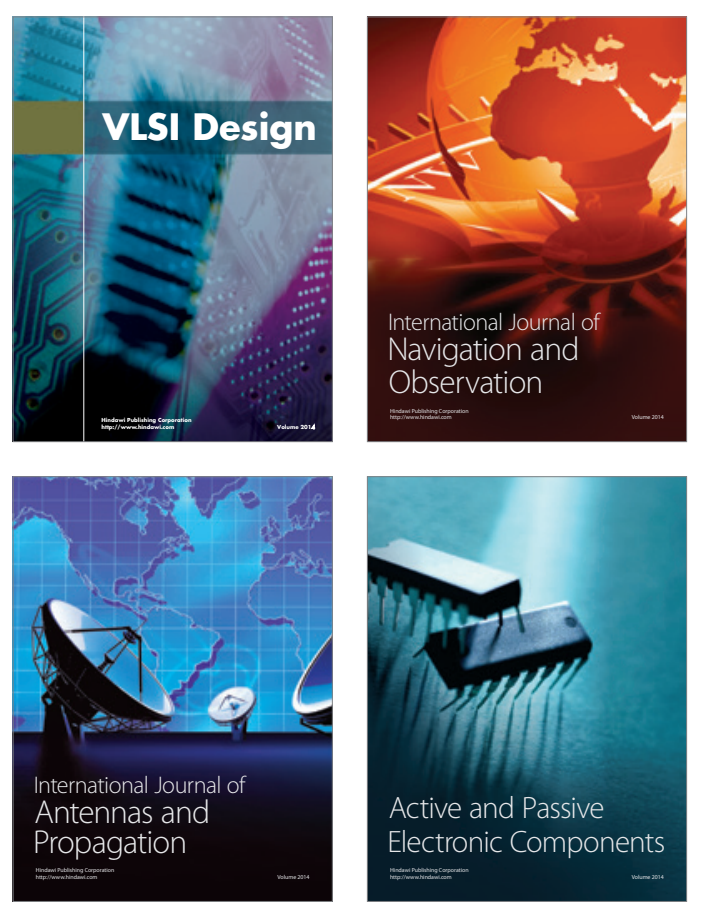
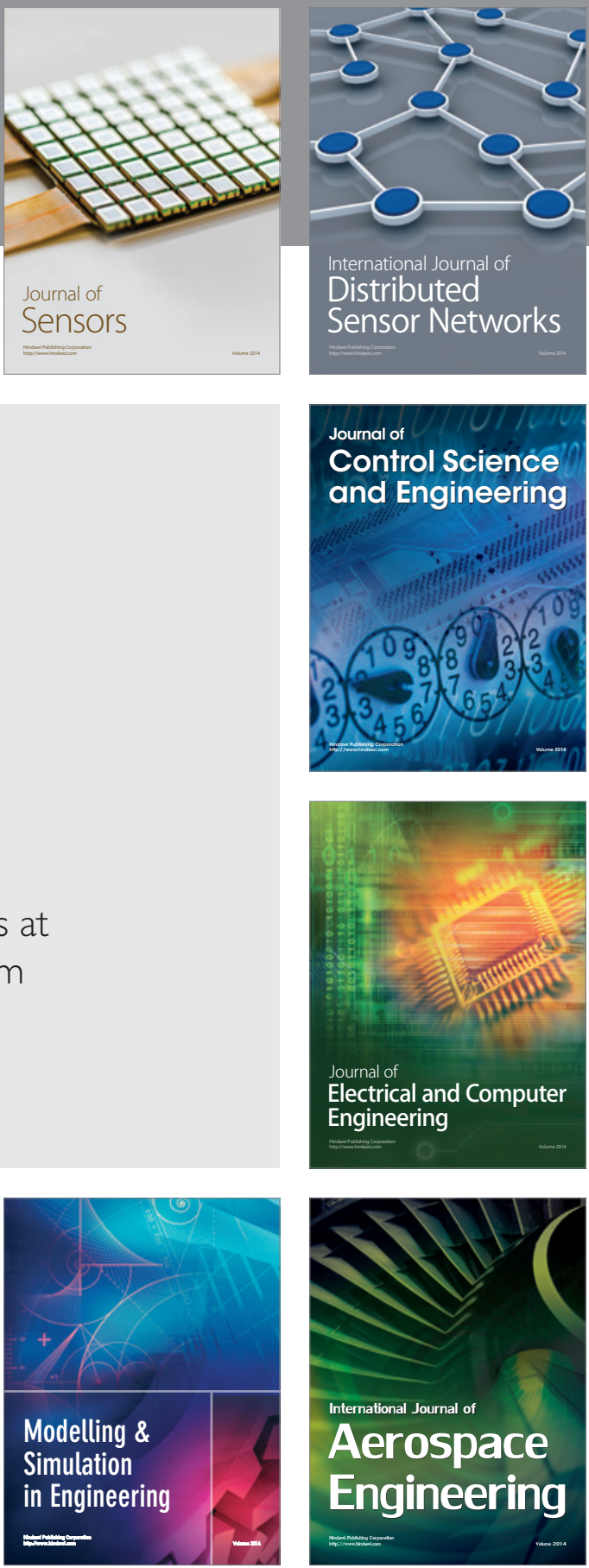

Journal of

Control Science

and Engineering
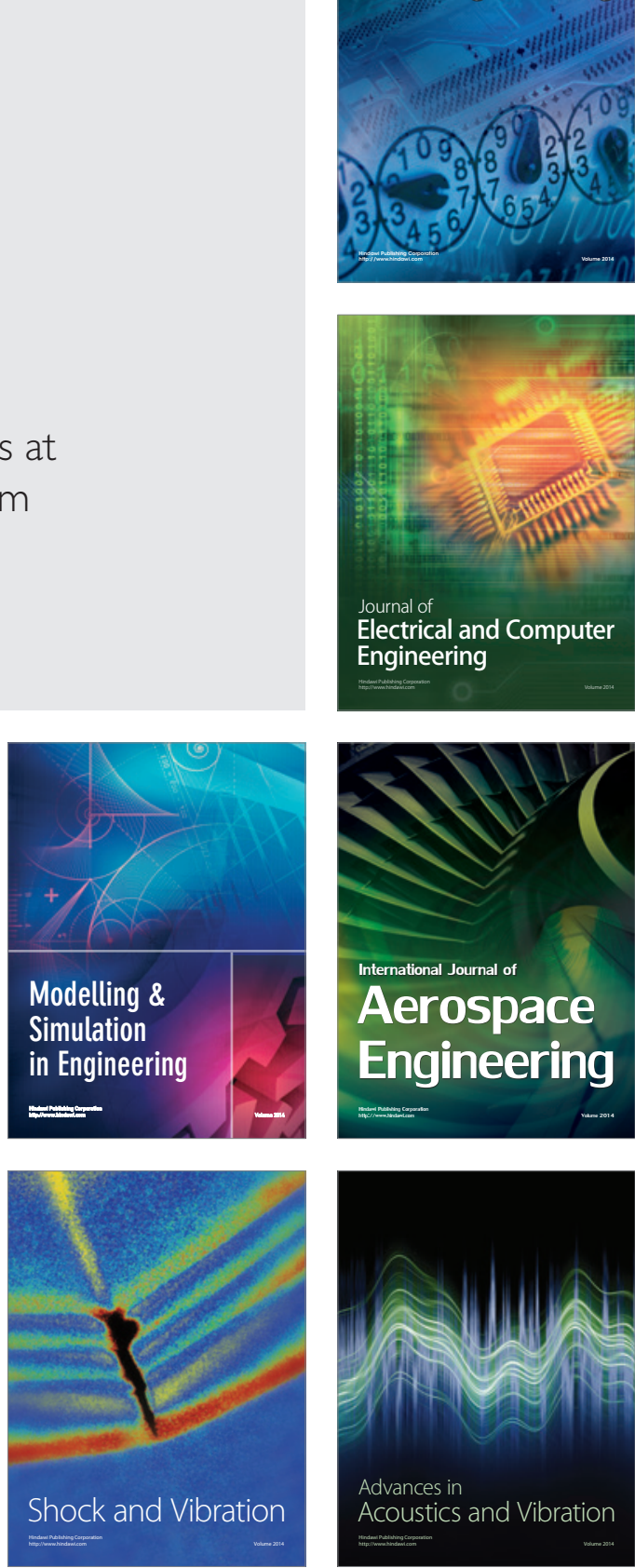